\title{
The parabolic Anderson model in a dynamic random environment: space-time ergodicity for the quenched Lyapunov exponent
}

\author{
D. Erhard ${ }^{1}$ \\ F. den Hollander ${ }^{2}$ \\ G. Maillard ${ }^{3}$
}

November 19, 2018

\begin{abstract}
We continue our study of the parabolic Anderson equation $\partial u(x, t) / \partial t=\kappa \Delta u(x, t)+$ $\xi(x, t) u(x, t), x \in \mathbb{Z}^{d}, t \geq 0$, where $\kappa \in[0, \infty)$ is the diffusion constant, $\Delta$ is the discrete Laplacian, and $\xi$ plays the role of a dynamic random environment that drives the equation. The initial condition $u(x, 0)=u_{0}(x), x \in \mathbb{Z}^{d}$, is taken to be non-negative and bounded. The solution of the parabolic Anderson equation describes the evolution of a field of particles performing independent simple random walks with binary branching: particles jump at rate $2 d \kappa$, split into two at rate $\xi \vee 0$, and die at rate $(-\xi) \vee 0$.

We assume that $\xi$ is stationary and ergodic under translations in space and time, is not constant and satisfies $\mathbb{E}(|\xi(0,0)|)<\infty$, where $\mathbb{E}$ denotes expectation w.r.t. $\xi$. Our main object of interest is the quenched Lyapunov exponent $\lambda_{0}(\kappa)=\lim _{t \rightarrow \infty} \frac{1}{t} \log u(0, t)$. In earlier work [6], 3] we established a number of basic properties of $\kappa \mapsto \lambda_{0}(\kappa)$ under certain mild space-time mixing and noisiness assumptions on $\xi$. In particular, we showed that the limit exists $\xi$-a.s., is finite and continuous on $[0, \infty)$, is globally Lipschitz on $(0, \infty)$, is not Lipschitz at 0 , and satisfies $\lambda_{0}(0)=\mathbb{E}(\xi(0,0))$ and $\lambda_{0}(\kappa)>\mathbb{E}(\xi(0,0))$ for $\kappa \in(0, \infty)$.

In the present paper we show that $\lim _{\kappa \rightarrow \infty} \lambda_{0}(\kappa)=\mathbb{E}(\xi(0,0))$ under an additional space-time mixing condition on $\xi$ we call Gärtner-hyper-mixing. This result, which completes our study of the quenched Lyapunov exponent for general $\xi$, shows that the parabolic Anderson model exhibits space-time ergodicity in the limit of large diffusivity. This fact is interesting because there are choices of $\xi$ that are Gärtner-hyper-mixing for which the annealed Lyapunov exponent $\lambda_{1}(\kappa)=\lim _{t \rightarrow \infty} \frac{1}{t} \log \mathbb{E}(u(0, t))$ is infinite on $[0, \infty)$, a situation that is referred to as strongly catalytic behavior. Our proof is based on a multiscale analysis of $\xi$, in combination with discrete rearrangement inequalities for local times of simple random walk and spectral bounds for discrete Schrödinger operators.
\end{abstract}

MSC 2010. Primary 60K35, 60H25, 82C44; Secondary 35B40, 60F10.

Key words and phrases. Parabolic Anderson equation, quenched Lyapunov exponent, large deviations, Gärtner-hyper-mixing, multiscale analysis, rearrangement inequalities, spectral bounds.

Acknowledgment. DE and FdH were supported by ERC Advanced Grant 267356 VARIS.

\footnotetext{
${ }^{1}$ Mathematical Institute, Leiden University, P.O. Box 9512, 2300 RA Leiden, The Netherlands, erhardd@math.leidenuniv.nl

${ }^{2}$ Mathematical Institute, Leiden University, P.O. Box 9512, 2300 RA Leiden, The Netherlands, denholla@math.leidenuniv.nl

${ }^{3}$ CMI-LATP, Aix-Marseille Université, 39 rue F. Joliot-Curie, F-13453 Marseille Cedex 13, France, maillard@cmi.univ-mrs.fr
} 


\section{Introduction and main theorem}

A fair amount is known about the behavior as a function of underlying parameters of the annealed Lyapunov exponents for the parabolic Anderson model in a dynamic random environment. For an overview we refer the reader to [5]. The main motivation behind the present paper is to understand the behavior of the quenched Lyapunov exponent, which is much harder to deal with. Our ultimate goal is to arrive at a full qualitative picture of the quenched Lyapunov exponent for general dynamic random environments subject to certain mild space-time mixing and noisiness assumptions.

Section 1.1 defines the parabolic Anderson model and recalls the main results from [6, 3]. Section 1.2 contains our main theorem, which states that the quenched Lyapunov exponent converges to the average value of the environment in the limit of large diffusivity. Section 1.3 contains definitions, whereas Section 1.4 discusses the main theorem, provides the necessary background, and gives a brief outline of the rest of the paper.

\subsection{Parabolic Anderson model}

The parabolic Anderson model is the partial differential equation

$$
\frac{\partial}{\partial t} u(x, t)=\kappa \Delta u(x, t)+\xi(x, t) u(x, t), \quad x \in \mathbb{Z}^{d}, t \geq 0 .
$$

Here, the $u$-field is $\mathbb{R}$-valued, $\kappa \in[0, \infty)$ is the diffusion constant, $\Delta$ is the discrete Laplacian acting on $u$ as

$$
\Delta u(x, t)=\sum_{\substack{y \in \mathbb{Z}^{d} \\\|y-x\|=1}}[u(y, t)-u(x, t)]
$$

$\left(\|\cdot\|\right.$ is the $l_{1}$-norm), while

$$
\xi=\left(\xi_{t}\right)_{t \geq 0} \text { with } \xi_{t}=\left\{\xi(x, t): x \in \mathbb{Z}^{d}\right\}
$$

is an $\mathbb{R}$-valued random field playing the role of a dynamic random environment that drives the equation. As initial condition for (1.1) we take

$$
u(x, 0)=u_{0}(x), x \in \mathbb{Z}^{d} \text {, with } u_{0} \text { non-negative, not identically zero, and bounded. }
$$

One interpretation of (1.1) and (1.4) comes from population dynamics. Consider the special case where $\xi(x, t)=\gamma \xi_{*}(x, t)-\delta$ with $\delta, \gamma \in(0, \infty)$ and $\xi_{*}$ an $\mathbb{N}_{0}$-valued random field. Consider a system of two types of particles, $A$ (catalyst) and $B$ (reactant), subject to:

- A-particles evolve autonomously according to a prescribed dynamics with $\xi_{*}(x, t)$ denoting the number of $A$-particles at site $x$ at time $t$;

- $B$-particles perform independent simple random walks at rate $2 d \kappa$ and split into two at a rate that is equal to $\gamma$ times the number of $A$-particles present at the same location at the same time;

- B-particles die at rate $\delta$;

- the average number of $B$-particles at site $x$ at time 0 is $u_{0}(x)$. 
Then

$$
\begin{aligned}
u(x, t)= & \text { the average number of } B \text {-particles at site } x \text { at time } t \\
& \text { conditioned on the evolution of the } A \text {-particles. }
\end{aligned}
$$

The $\xi$-field is defined on a probability space $(\Omega, \mathcal{F}, \mathbb{P})$. Throughout the paper we assume that

- $\xi$ is stationary and ergodic under translations in space and time.

- $\xi$ is not constant and $\mathbb{E}(|\xi(0,0)|)<\infty$.

The formal solution of (1.1) is given by the Feynman-Kac formula

$$
u(x, t)=E_{x}\left(\exp \left\{\int_{0}^{t} \xi\left(X^{\kappa}(s), t-s\right) d s\right\} u_{0}\left(X^{\kappa}(t)\right)\right)
$$

where $X^{\kappa}=\left(X^{\kappa}(t)\right)_{t \geq 0}$ is the continuous-time simple random walk jumping at rate $2 d \kappa$ (i.e., the Markov process with generator $\kappa \Delta)$, and $P_{x}$ is the law of $X^{\kappa}$ when $X^{\kappa}(0)=x$. In [3] we proved the following:

(0) Subject to the assumption that $\xi$-a.s. $s \mapsto \xi(x, s)$ is locally integrable for every $x$ and that $\mathbb{E}\left(e^{q \xi(0,0)}\right)<\infty$ for all $q \geq 0$, (1.7) is finite for all $x, t$ and is the solution of (1.1).

The quenched Lyapunov exponent associated with (1.1) is defined as

$$
\lambda_{0}(\kappa)=\lim _{t \rightarrow \infty} \frac{1}{t} \log u(0, t) .
$$

In [6] we showed that $\lambda_{0}(0)=\mathbb{E}(\xi(0,0))$ and $\lambda_{0}(\kappa)>\mathbb{E}(\xi(0,0))$ for $\kappa \in(0, \infty)$ as soon as the limit in (1.8) exists. In [3] we proved the following:

(1) Subject to certain space-time mixing assumptions on $\xi$, the limit in (1.8) exists $\xi$-a.s. and in $L^{1}(\mathbb{P})$, is $\xi$-a.s. constant, is finite, and does not depend on $u_{0}$ satisfying (1.4).

(2) Subject to certain additional noisiness assumptions on $\xi, \kappa \mapsto \lambda_{0}(\kappa)$ is continuous on $[0, \infty)$, is globally Lipschitz on $(0, \infty)$, and is not Lipschitz at 0 .

\subsection{Main theorem and examples}

Our main result is the following.

Theorem 1.1. If $u_{0}=\delta_{0}$ and $\xi$ is Gärtner-hyper-mixing, then

$$
\lim _{\kappa \rightarrow \infty} \lambda_{0}(\kappa)=\mathbb{E}(\xi(0,0))
$$

The definition of Gärtner-hyper-mixing is given in Definitions 1.31.5 below. A weaker form of these definitions was introduced and exploited in [3]. Here are two examples of $\xi$-fields that are Gärtner-hyper-mixing.

Example 1.2. (See [3])

(e1) Let $Y=\left(Y_{t}\right)_{t \geq 0}$ be a stationary and ergodic $\mathbb{R}$-valued Markov process satisfying

$$
\mathbb{E}\left[e^{q \sup _{t \in[0,1]}\left|Y_{t}\right|}\right]<\infty \quad \forall q \geq 0 .
$$


Let $(Y(x))_{x \in \mathbb{Z}^{d}}$ be a field of independent copies of $Y$. Then $\xi$ given by $\xi(x, t)=Y_{t}(x)$ is Gärtner-hyper-mixing.

(e2) Let $\xi$ be the zero-range process with rate function $g: \mathbb{N}_{0} \rightarrow(0, \infty)$ given by $g(k)=k^{\beta}$, $\beta \in(0,1]$, and transition probabilities given by simple random walk on $\mathbb{Z}^{d}$. If $\xi$ starts from the product measure $\pi_{\rho}, \rho \in(0, \infty)$, with marginals

$$
\forall x \in \mathbb{Z}^{d}: \quad \pi_{\rho}\left\{\eta \in \mathbb{N}_{0}^{\mathbb{Z}^{d}}: \eta(x)=k\right\}= \begin{cases}\gamma \frac{\rho^{k}}{\prod_{l=1}^{k} g(l)}, & \text { if } k>0, \\ \gamma, & \text { if } k=0,\end{cases}
$$

where $\gamma \in(0, \infty)$ is a normalization constant, then $\xi$ is Gärtner-hyper-mixing.

(The proof in [3] is without the supremum in (1.15) below, but easily carries over by inspection.) Example (e1) includes independent spin-flips, example (e2) includes independent random walks.

We expect that most interacting particle systems are Gärtner-hyper-mixing, including such classical systems as the stochastic Ising model, the contact process, the voter model and the exclusion process. Since these are bounded random fields, conditions (a2) and (a3) in Definition 1.5 below are redundant and only condition (a1) needs to be verified. Note that the constant $\delta$ in (1.15) below was allowed to be choosen arbitrarily large in [3. However, in this work we assume that $\delta$ goes to zero in a certain way (see Definition (1.5)) so that (a1) indeed becomes an issue. We will not tackle the problem of solving this issue for the above mentioned fields in the present paper.

\subsection{Definitions}

Throughout the rest of this paper we assume without loss of generality that $\mathbb{E}(\xi(0,0))=0$.

For $a_{1}, a_{2}, N \in \mathbb{N}$, denote by $\Delta_{N}\left(a_{1}, a_{2}\right)$ the set of $\left(\mathbb{Z}^{d} \times \mathbb{N}\right)$-valued sequences $\left\{\left(x_{i}, k_{i}\right)\right\}_{i=1}^{N}$ that are increasing with respect to the lexicographic ordering of $\mathbb{Z}^{d} \times \mathbb{N}$ and are such that, for all $1 \leq i<j \leq N$,

$$
x_{j} \equiv x_{i}\left(\bmod a_{1}\right), \quad k_{j} \equiv k_{i}\left(\bmod a_{2}\right) .
$$

For $A \geq 1, \alpha>0, R \in \mathbb{N}, x \in \mathbb{Z}^{d}$ and $k, b, c \in \mathbb{N}_{0}$, define the space-time blocks (see Fig. 11)

$$
\tilde{B}_{R}^{A, \alpha}(x, k ; b, c)=\left(\prod_{j=1}^{d}\left[(x(j)-1-b) \alpha A^{R},(x(j)+1+b) \alpha A^{R}\right) \cap \mathbb{Z}^{d}\right) \times\left[(k-c) A^{R},(k+1) A^{R}\right) .
$$

Abbreviate $B_{R}^{A, \alpha}(x, k)=\tilde{B}_{R}^{A, \alpha}(x, k ; 0,0)$ and $B_{R}^{A}(x, k)=B_{R}^{A, 1}(x, k)$, and define the spaceblocks

$$
Q_{R}^{A, \alpha}(x)=x+\left[0, \alpha A^{R}\right)^{d} \cap \mathbb{Z}^{d} .
$$

Definition 1.3. [Good and bad blocks]

For $A \geq 1, \alpha>0, R \in \mathbb{N}, x \in \mathbb{Z}^{d}, m>0, k \in \mathbb{N}_{0}, \delta \in[0$, ess $\sup [\xi(0,0)]]$ and $b, c \in \mathbb{N}_{0}$, the $R$-block $B_{R}^{A, \alpha}(x, k)$ is called $(\delta, b, c)$-good for the potential $\xi$ when, for all $s \in\left[(k-c) A^{R},(k+\right.$ 1) $\left.A^{R}-1 / m\right)$,

$$
\frac{1}{\left|Q_{R}^{A, \alpha}(y)\right|} \sum_{z \in Q_{R}^{A, \alpha}(y)} \sup _{r \in[s, s+1 / m)} \xi(z, r) \leq \delta \quad \forall y \in \mathbb{Z}^{d}: Q_{R}^{A, \alpha}(y) \times\{s\} \subseteq \tilde{B}_{R}^{A, \alpha}(x, k ; b, c),
$$

and is called $(\delta, b, c)$-bad otherwise. 


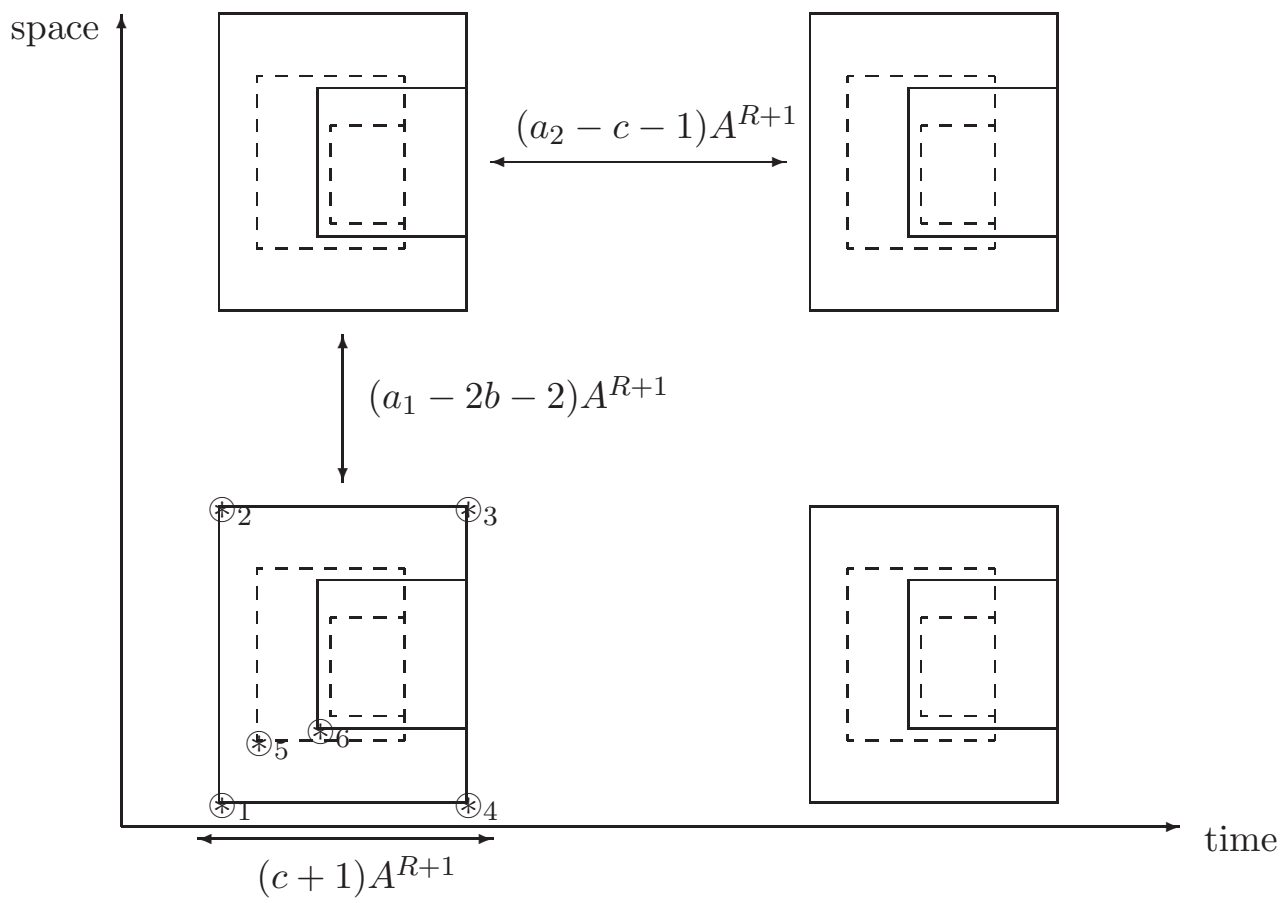

Figure 1: The dashed blocks are $R$-blocks, i.e., $B_{R}^{A}(x, k)$ (inner) and $\tilde{B}_{R}^{A}(x, k ; b, c)$ (outer) for some choice of $A, x, k, b, c$. The solid blocks are $(R+1)$-blocks, i.e., $B_{R+1}^{A}(y, l)$ (inner) and $\tilde{B}_{R+1}^{A}(y, l ; b, c)$ (outer) for some choice of $A, y, l, b, c$ such that these $(R+1)$-blocks contain the corresponding $R$ blocks. All these blocks belong to the same equivalence class. The symbols $\left\{\circledast_{i}\right\}_{i=1,2,3,4,5,6}$ represents the space-time coordinates $\circledast_{1}=\left((y-1-b) A^{R+1},(l-c) A^{R+1}\right), \circledast_{2}=\left((y+1+b) A^{R+1},(l-c) A^{R+1}\right)$, $\circledast_{3}=\left((y+1+b) A^{R+1},(l+1) A^{R+1}\right), \circledast_{4}=\left((y-1-b) A^{R+1},(l+1) A^{R+1}\right), \circledast_{5}=\left((x-1-b) A^{R},(k-c) A^{R}\right)$, $\circledast_{6}=\left((y-1) A^{R+1}, l A^{R+1}\right)$.

For $A \geq 1, \alpha>0, R \in \mathbb{N}, x \in \mathbb{Z}^{d}, m>0, k \in \mathbb{N}_{0}, \delta \in[0$, ess $\sup [\xi(0,0)]]$ and $b, c \in \mathbb{N}_{0}$, let

$$
\begin{aligned}
& \mathcal{A}_{R}^{A, \alpha, \delta, m}(x, k ; b, c) \\
& =\left\{B_{R+1}^{A, \alpha}(x, k) \text { is }(\delta, b, c) \text {-good, but contains an } R \text {-block that is }(\delta, b, c) \text {-bad }\right\} .
\end{aligned}
$$

\section{Definition 1.4. [Gärtner-mixing]}

The $\xi$-field is called $(A, \alpha, \delta, m, b, c)$-Gärtner-mixing when there are $a_{1}, a_{2} \in \mathbb{N}$ such that

$$
\sup _{\left(x_{i}, k_{i}\right)_{i=1}^{N} \in \Delta_{N}\left(a_{1}, a_{2}\right)} \mathbb{P}\left(\bigcap_{i=1}^{N} \mathcal{A}_{R}^{A, \alpha, \delta, m}\left(x_{i}, k_{i} ; b, c\right)\right) \leq\left(A^{-4 d(2 d+1)(d+1) R}\right)^{N} \quad \forall R \in \mathbb{N}, N \in \mathbb{N} .
$$

\section{Definition 1.5. [Gärtner-hyper-mixing]}

The $\xi$-field is called Gärtner-hyper-mixing when the following conditions are satisfied:

(a1) There are $b, c \in \mathbb{N}_{0}$ and $K \geq 0$ such that for every $\delta>0$ there are $A_{0}>1$ and $m_{0}>0$ such that $\xi \mathbb{1}\{\xi \geq K\}$ and $\xi$ are $(A, \alpha, \delta, m, b, c)$-Gärtner-mixing for all $A \geq A_{0}, m \geq m_{0}$ and all $\alpha \geq 1$, with $a_{1}, a_{2}$ in Definition 1.4 not depending on $A, m$ and $\alpha$.

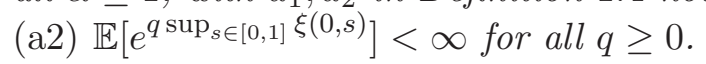

(a3) There are $R_{0} \in \mathbb{N}$ and $C_{1} \in[0$, ess $\sup [\xi(0,0)]]$ such that

$$
\mathbb{P}\left(\sup _{s \in[0,1]} \frac{1}{\left|B_{R}\right|} \sum_{y \in B_{R}} \xi(y, s) \geq C\right) \leq\left|B_{R}\right|^{-\alpha} \quad \forall R \geq R_{0}, C \geq C_{1},
$$

for some $\alpha>[2 d(2 d+1)+1](d+2) / d$, where $B_{R}=[-R, R]^{d} \cap \mathbb{Z}^{d}$. 


\subsection{Discussion}

1. What is interesting about Theorem 1.1 is that it reveals a sharp contrast with what is known for the annealed Lyapunov exponent

$$
\lambda_{1}(\kappa)=\lim _{t \rightarrow \infty} \frac{1}{t} \log \mathbb{E}(u(0, t)) .
$$

Indeed, there are choices of $\xi$ for which $\kappa \mapsto \lambda_{1}(\kappa)$ is everywhere infinite on [0, $\left.\infty\right)$, a property referred to as strongly catalytic behavior. For instance, as shown in [4], if $\xi$ is $\gamma$ times a field of independent simple random walks starting in a Poisson equilibrium with arbitrary density, then this uniform divergence occurs in $d=1,2$ for $\gamma \in(0, \infty)$ and in $d \geq 3$ for $\gamma \in\left[1 / G_{d}, \infty\right)$, with $G_{d}$ the Green function of simple random walk at the origin. By Example 1.2)(e2) (with $\beta=1$ ), this choice of $\xi$ is Gärtner-hyper-mixing.

2. The annealed Lyapunov exponents

$$
\lambda_{p}(\kappa)=\lim _{t \rightarrow \infty} \frac{1}{t} \log \mathbb{E}\left([u(0, t)]^{p}\right), \quad p \in \mathbb{N},
$$

were studied in detail in a series of papers where $\xi$ was chosen to evolve according to four specific interacting particle systems in equilibrium: independent Brownian motions, independent simple random walks, the simple symmetric exclusion process, and the voter model (for an overview, see [5]). Their behavior turns out to be very different from that of $\lambda_{0}(\kappa)$. In [3] it was conjectured that

$$
\lim _{\kappa \rightarrow \infty}\left[\lambda_{p}(\kappa)-\lambda_{0}(\kappa)\right]=0 \quad \forall p \in \mathbb{N}
$$

because $\xi$ is ergodic in space and time. For the case where $\lambda_{p}(\kappa) \equiv \infty$ this statement is to be read as saying that $\lim _{\kappa \rightarrow \infty} \lambda_{0}(\kappa)=\infty$. Theorem 1.1 shows that this conjecture is false and that, for $\xi$ Gärtner-hyper-mixing and satisfying conditions (0) and (2) in Section [1.1, the qualitative behavior of $\kappa \mapsto \lambda_{0}(\kappa)$ is as in Fig. 2.

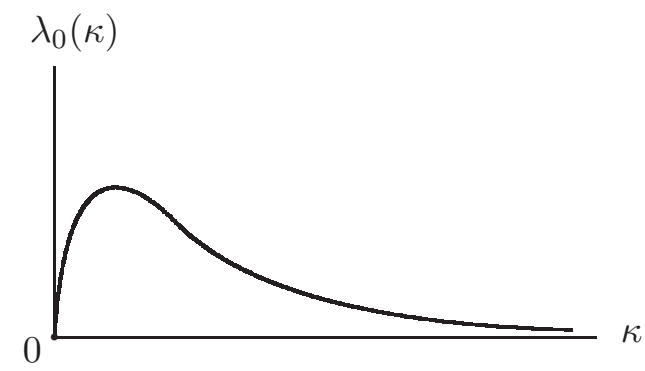

Figure 2: Qualitative behavior of $\kappa \mapsto \lambda_{0}(\kappa)$.

3. Our proof of Theorem 1.1 is based on a multiscale analysis of $\xi$, in the spirit of [10] and consists of two major steps:

(I) We look at the bad $R$-blocks for all $R \in \mathbb{N}$. First we show that bad $R$-blocks are rare for large $R$. Next, using a discrete rearrangement inequality for local times of simple random walk, we show that the contribution to the expectation in (1.7) coming from bad $R$-blocks increases when we move them towards the origin. Therefore this contribution can be bounded from above by an expectation that pretends the bad $R$-blocks to be 
rearranged in a space-time cylinder around the origin. Since bad $R$-blocks are rare, this cylinder is narrow. Afterwards, because simple random walk is unlikely to spend a lot of time in narrow space-time cylinder, we are able to control the contribution coming from bad $R$-blocks to the expectation (1.7) uniformly in $t$ and $\kappa$.

(II) We look at the good $R$-blocks for all $R \in \mathbb{N}$. We control their contribution by using an eigenvalue expansion of (1.7). An analysis of the largest eigenvalue in this expansion concludes the argument.

The remainder of this paper is organized as follows. In Section 2 we formulate three key propositions and use these to prove Theorem 1.1. The three propositions are proved in Sections 3 7, respectively. In Appendix A we prove two technical lemmas that are needed in Section 4, while in Appendix B we prove a spectral bound that is needed in Section 7 .

\section{Three key propositions and proof of Theorem 1.1}

To state our three key propositions we need some definitions. Fix $k_{*} \in \mathbb{N}$, and $t>0$. We say that $\Phi:[0, t] \rightarrow \mathbb{Z}^{d}$ is a path when

$$
\|\Phi(s)-\Phi(s-)\| \leq 1 \quad \forall s \in[0, t] .
$$

Define the set of paths

$$
\Pi\left(k_{*}, t, A\right)=\left\{\Phi:[0, t] \rightarrow \mathbb{Z}^{d}: \Phi \text { crosses } k_{*} \text { 1-blocks }\right\} .
$$

For $B_{R} \subseteq \mathbb{Z}^{d} \times[0, t]$, let $l_{t}\left(B_{R}\right)$ denote the local time of $X^{\kappa}$ in $B_{R}$ up to time $t$, and $l_{t}\left(\pi_{1}\left(B_{R}\right)\right)$ the local time of $\pi_{1}\left(X^{\kappa}\right)$ (the first coordinate of $\left.X^{\kappa}\right)$ in $\pi_{1}\left(B_{R}\right)$ up to time $t$. Furthermore, let $l_{t}\left(\mathrm{BAD}_{R}^{\delta}\left(\xi_{K}\right)\right)$ and $l_{t}\left(\mathrm{BAD}^{\delta}(\xi)\right)$ denote the local time of $X^{\kappa}$ in $(\delta, b, c)$-bad $R$-blocks up to time $t$ for the potential $\xi \mathbb{1}\{\xi \geq K\}$ and in $(\delta, b, c)$-bad 1-blocks up to time $t$ for the potential $\xi$, respectively. Here and in the rest of the paper a bad $R$-block is $(\delta, b, c)$-bad for a choice of $K, \delta, b, c$ and some $A \geq A_{0}, m \geq m_{0}$, according to Definition 1.5.

In what follows, when we write sums like $\sum_{0 \leq k<t / A^{R}}$ or $\sum_{R=1}^{\varepsilon \log t}$ we will pretend that $t / A^{R}$ and $\varepsilon \log t$ are integer in order not to burden the notation with round off brackets. From the context it will always be clear where to place the brackets.

Proposition 2.1. There is a $C_{2}>0$ such that for every $\varepsilon>0$ and $\delta>0$ there is an $A=A(\varepsilon)>3$, satisfying $\lim _{\varepsilon \downarrow 0} A(\varepsilon, \delta)=\infty$, such that $\xi$-a.s. for all $\kappa>0$ and all $t>0$ large enough,

$$
\begin{gathered}
E_{0}\left(\exp \left\{\int_{0}^{t} \xi\left(X^{\kappa}(s), t-s\right) d s\right\}\right) \leq e^{-t}+\left[E_{0}\left(\exp \left\{\int_{0}^{t} \bar{\xi}\left(X^{\kappa}(s), t-s\right) d s\right\}\right)^{1 / 2}\right. \\
\times E_{0}\left(\exp \left\{2 \delta A^{d} l_{t}\left(\mathrm{BAD}^{\delta}(\xi)\right)+2 \sum_{R=1}^{\varepsilon \log t} \delta A^{(R+1) d} l_{t}\left(\mathrm{BAD}_{R}^{\delta}\left(\xi_{K}\right)\right)\right\}\right. \\
\left.\left.\times \mathbb{1}\left\{\exists k_{*} \leq C_{2} \kappa t: X^{\kappa} \in \Pi\left(k_{*}, t, A\right)\right\}\right)^{1 / 2}\right]
\end{gathered}
$$

where

$$
\bar{\xi}(x, s)=2 \xi(x, s) \mathbb{1}\left\{\xi(x, s)<\delta A^{d},(x, s) \text { is in a good 1-block of } \xi\right\} \text {. }
$$


Proposition 2.2. There is a $C_{2}>0$ such that for every $\varepsilon, \tilde{\varepsilon}>0$ and $\delta>0$ there is an $A=A(\varepsilon, \tilde{\varepsilon}, \delta)>3$, satisfying $\lim _{\tilde{\varepsilon} \downarrow 0} A(\varepsilon, \tilde{\varepsilon}, \delta)=\infty$, such that $\xi$-a.s. for all $\kappa>0$ and all $t>0$ large enough,

$$
\begin{array}{r}
E_{0}\left(\exp \left\{2 \delta A^{d} l_{t}\left(\mathrm{BAD}^{\delta}(\xi)\right)+2 \sum_{R=1}^{\varepsilon \log t} \delta A^{(R+1) d} l_{t}\left(\mathrm{BAD}_{R}^{\delta}\left(\xi_{K}\right)\right)\right\}\right. \\
\left.\times \mathbb{1}\left\{\exists k_{*} \leq C_{2} \kappa t: X^{\kappa} \in \Pi\left(k_{*}, t, A\right)\right\}\right) \leq e^{\tilde{\varepsilon} t}
\end{array}
$$

Proposition 2.3. There is a constant $C_{3}>0$ such that, for every $A>1$ and $\delta>0$,

$$
\limsup _{\kappa \rightarrow \infty} \limsup _{n \rightarrow \infty} \frac{1}{A n} \log E_{0}\left(\exp \left\{\int_{0}^{A n} \bar{\xi}\left(X^{\kappa}(s), A n-s\right) d s\right\}\right) \leq \frac{C_{3}}{A}+4 \delta \quad \xi-\text { a.s. }
$$

Proposition 2.1 estimates the Feynman-Kac formula in (1.7) in terms of bad blocks and good blocks, Proposition 2.2 controls the contribution of bad block, while Proposition 2.3 controls the contribution of good blocks.

We are now ready to prove Theorem 1.1 .

Proof. Note that by Theorem 1.2(i) in [6], for all $\kappa \geq 0$ we have the lower bound $\lambda_{0}(\kappa) \geq 0$. Thus, it suffices to show the inequality in the reverse direction. To that end, fix $C_{2}, C_{3}>0$ according to Propositions 2.1 2.3, and fix $\varepsilon, \tilde{\varepsilon}, \delta>0$. According to Proposition 2.2, there is an $A=A(\varepsilon, \tilde{\varepsilon}, \delta)$ such that, $\xi$-a.s. for all $\kappa>0$ and all $t$ of the form $t=A n$ with $n \in \mathbb{N}$ large enough, the term in the left-hand side of (2.5) is bounded from above by $e^{\tilde{\varepsilon} A n}$. According to Proposition 2.3, we have

$$
E_{0}\left(\exp \left\{\int_{0}^{A n} \bar{\xi}\left(X^{\kappa}(s), A n-s\right) d s\right\}\right) \leq e^{4 \delta A n+C_{3} n+\chi(\kappa, n)}
$$

with $\lim \sup _{\kappa \rightarrow \infty} \lim \sup _{n \rightarrow \infty} \chi(\kappa, n) / n=0$. Proposition 2.1 therefore yields that, for all $\varepsilon, \tilde{\varepsilon}, \delta>0$,

$$
\limsup _{\kappa \rightarrow \infty} \lambda_{0}(\kappa) \leq \frac{C_{3}}{2 A}+2 \delta+\frac{\tilde{\varepsilon}}{2} .
$$

Since $\lim _{\tilde{\varepsilon} \downarrow 0} A(\varepsilon, \tilde{\varepsilon}, \delta)=\infty$ by Proposition 2.2 , we get that for all $\delta>0$,

$$
\limsup _{\kappa \rightarrow \infty} \lambda_{0}(\kappa) \leq 2 \delta
$$

Let $\delta \downarrow 0$ to get the claim.

\section{Proof of Proposition 2.1}

The proof is given in Section 3.1 subject to Lemmas 3.13 .2 below. The proof of these lemmas is given in Section 3.2 , 


\subsection{Proof of Proposition 2.1 subject to two lemmas}

For $A \geq 1, R \in \mathbb{N}$ and $\Phi \in \Pi\left(k_{*}, t, A\right)$, define

$$
\begin{aligned}
\Xi_{R}^{A}(\Phi) & =\text { number of bad } R \text {-blocks crossed by } \Phi, \\
\Xi_{R}^{A, k_{*}} & =\sup _{\Phi \in \Pi\left(k_{*}, t, A\right)} \Xi_{R}^{A}(\Phi) .
\end{aligned}
$$

Lemma 3.1. For every $\varepsilon>0$ there is an $A=A(\varepsilon)>3$ satisfying $\lim _{\varepsilon \downarrow 0} A(\varepsilon)=\infty$ such that

$$
\mathbb{P}\left(\Xi_{R}^{A, k_{*}}>0 \text { for some } R \geq \varepsilon \log t \text { and some } k_{*} \in \mathbb{N}\right)
$$

is summable over $t \in \mathbb{N}$. A possible choice is $A=e^{1 / a \varepsilon[2 d(2 d+1)+1]}$ for some $a>1$.

Lemma 3.2. There is a $C_{2}>0$ such that $\xi$-a.s. for all $A>1$, all $t>0$ and all $\kappa>0$ large enough,

$$
E_{0}\left(\exp \left\{\int_{0}^{t} \xi\left(X^{\kappa}(s), t-s\right) d s\right\} \mathbb{1}\left\{\exists k_{*}>C_{2} \kappa t: X^{\kappa} \in \Pi\left(k_{*}, t, A\right)\right\}\right) \leq e^{-t} .
$$

We are now ready to prove Proposition 2.1.

Proof. Fix $C_{2}$ in accordance with Lemma 3.2 and $\varepsilon>0$. Let $\delta>0$ and fix $A>1$ according to Lemma 3.1 such that $\delta A^{d} \geq K$, see Definition 1.5. Note that

$$
\begin{gathered}
E_{0}\left(\exp \left\{\int_{0}^{t} \xi\left(X^{\kappa}(s), t-s\right) d s\right\} \mathbb{1}\left\{\exists k_{*} \leq C_{2} \kappa t: X^{\kappa} \in \Pi\left(k_{*}, t, A\right)\right\}\right) \\
=E_{0}\left(\exp \left\{\sum_{i=1}^{N\left(X^{\kappa}, t\right)} \int_{s_{i-1}}^{s_{i}} \xi\left(x_{i-1}, t-u\right) d u+\int_{s_{N\left(X^{\kappa}, t\right)}}^{t} \xi\left(x_{N\left(X^{\kappa}, t\right)}, t-u\right) d u\right\}\right. \\
\left.\times \mathbb{1}\left\{\exists k_{*} \leq C_{2} \kappa t: X^{\kappa} \in \Pi\left(k_{*}, t, A\right)\right\}\right),
\end{gathered}
$$

where $N\left(X^{\kappa}, t\right)$ is the number of jumps by $X^{\kappa}$ up to time $t, 0=x_{0}, x_{1}, \ldots, x_{N\left(X^{\kappa}, t\right)}$ are the nearest-neighbor sites visited, and $0=s_{0}<s_{1}<\cdots<s_{N\left(X^{\kappa}, t\right)} \leq t$ are the jump times. To analyze (3.4), define

$$
\begin{aligned}
\Lambda_{t}\left(\mathrm{BAD}_{R}^{\delta}\right)=\bigcup_{i=1}^{N\left(X^{\kappa}, t\right)}\left\{u \in\left[s_{i-1}, s_{i}\right): \delta A^{R d}<\xi\left(x_{i-1}, t-u\right) \leq \delta A^{(R+1) d}\right\} \\
\bigcup\left\{u \in\left[s_{N\left(X^{\kappa}, t\right)}, t\right): \delta A^{R d}<\xi\left(x_{N\left(X^{\kappa}, t\right)}, t-u\right) \leq \delta A^{(R+1) d}\right\} .
\end{aligned}
$$

Then the contribution to the exponential in (3.4) may be bounded from above by

$$
\int_{0}^{t} \xi\left(X^{\kappa}(s), t-s\right) \mathbb{1}\left\{\xi\left(X^{\kappa}(s), t-s\right)<\delta A^{d}\right\} d s+\sum_{R \in \mathbb{N}} \delta A^{(R+1) d}\left|\Lambda_{t}\left(\mathrm{BAD}_{R}^{\delta}\right)\right|,
$$

By Definition 1.3 and the fact that $\delta A^{d} \geq K$ (see the line preceding (3.4)), if $\delta A^{R d}<\xi\left(x_{i-1}, t-\right.$ $u) \leq \delta A^{(R+1) d}$, then $\left(x_{i-1}, t-u\right)$ belongs to a bad $R$-block for the potential $\xi \mathbb{1}\{\xi \geq K\}$. Hence

$$
\left|\Lambda_{t}\left(\mathrm{BAD}_{R}^{\delta}\right)\right| \leq l_{t}\left(\mathrm{BAD}_{R}^{\delta}\left(\xi_{K}\right)\right)
$$


To continue we write the indicator in (3.5)

$$
\begin{aligned}
& \mathbb{1}\left\{\xi\left(X^{\kappa}(s), t-s\right)<\delta A^{d},\left(X^{\kappa}(s), t-s\right) \text { is in a good 1-block of } \xi\right\} \\
& +\mathbb{1}\left\{\xi\left(X^{\kappa}(s), t-s\right)<\delta A^{d},\left(X^{\kappa}(s), t-s\right) \text { is in a bad 1-block of } \xi\right\}
\end{aligned}
$$

By Lemma 3.1 and our choice of $A$ at the beginning of the proof, $\xi$-a.s. for $t$ large enough there are no bad $R$-blocks with $R>\varepsilon \log t$. Thus, the expectation in the right-hand side of (3.4) may be estimated from above by

$$
\begin{aligned}
E_{0}\left(\operatorname { e x p } \left\{\int_{0}^{t} \xi\left(X^{\kappa}(s), t-s\right)\right.\right. \\
\left.\quad \times \mathbb{1}\left\{\xi\left(X^{\kappa}(s), t-s\right)<\delta A^{d},\left(X^{\kappa}(s), t-s\right) \text { is in a good 1-block of } \xi\right\} d s\right\} \\
\quad \times \exp \left\{\delta A^{d} l_{t}\left(\mathrm{BAD}^{\delta}(\xi)\right)+\sum_{R=1}^{\varepsilon \log t} \delta A^{(R+1) d} l_{t}\left(\mathrm{BAD}_{R}^{\delta}\left(\xi_{K}\right)\right)\right\} \\
\left.\quad \times \mathbb{1}\left\{\exists k_{*} \leq C_{2} \kappa t: X^{\kappa} \in \Pi\left(k_{*}, t, A\right)\right\}\right) .
\end{aligned}
$$

Recall (2.4). An application of the Cauchy-Schwarz inequality yields the following upper bound for (3.9):

$$
\begin{aligned}
& E_{0}\left(\exp \left\{\int_{0}^{t} \bar{\xi}\left(X^{\kappa}(s), t-s\right) d s\right\}\right)^{1 / 2} \\
& \times E_{0}\left(\exp \left\{2 \delta A^{d} l_{t}\left(\mathrm{BAD}^{\delta}(\xi)\right)+2 \sum_{R=1}^{\varepsilon \log t} \delta A^{(R+1) d} l_{t}\left(\mathrm{BAD}_{R}^{\delta}\left(\xi_{K}\right)\right)\right\}\right. \\
& \left.\times \mathbb{1}\left\{\exists k_{*} \leq C_{2} \kappa t: X^{\kappa} \in \Pi\left(k_{*}, t, A\right)\right\}\right)^{1 / 2} .
\end{aligned}
$$

The claim in (2.3) therefore follows by combining (3.4), (3.6 3.7) and (3.9)-(3.10) with Lemma 3.2.

\subsection{Proof of Lemmas $3 . 1 \longdiv { 3 . 2 }$}

Proof. For the proof of Lemma 3.1, see [3, Lemma 3.3]. To prove Lemma 3.2, use CauchySchwarz to estimate the expectation in (3.3) from above by

$$
\left[E_{0}\left(\exp \left\{2 \int_{0}^{t} \xi\left(X^{\kappa}(s), t-s\right) d s\right\}\right)\right]^{1 / 2}\left[P_{0}\left(\exists k_{*}>C_{2} \kappa t: X^{\kappa} \in \Pi\left(k_{*}, t, A\right)\right)\right]^{1 / 2} .
$$

To bound the first term in (3.11), note that by [3. Eq.(3.54)] there is a $C>0$ such that $\xi$-a.s. for all $t, \kappa>0$,

$$
E_{0}\left(e^{2 \int_{0}^{t} \xi\left(X^{\kappa}(s), t-s\right) d s}\right) \leq e^{t C(\kappa+1)}
$$


To bound the second term in (3.11) we use a similar strategy as for the proof of Lemma 4.4, Given $l_{1}, \ldots, l_{t / A} \in \mathbb{N}$, we say that $X^{\kappa}$ has label $\left(l_{1}, \ldots, l_{t / A}\right)$ when $X^{\kappa}$ crosses $l_{i}$ 1-blocks in the time interval $[(i-1) A, i A), i \in\{1, \ldots, t / A\}$. Fix $C_{2}>0$ and write

$$
\begin{aligned}
& P_{0}\left(X^{\kappa} \in \Pi\left(k_{*}, t, A\right) \text { for some } k_{*}>C_{2} \kappa t\right) \\
& =\sum_{j=1}^{\infty} P_{0}\left(X^{\kappa} \in \Pi\left(k_{*}, t, A\right) \text { for some } k_{*} \in\left(j C_{2} \kappa t,(j+1) C_{2} \kappa t\right]\right) .
\end{aligned}
$$

For $j \in \mathbb{N}$, write $\sum_{\left(l_{1}^{j}, \ldots, l_{t / A}^{j}\right)}$ to denote the sum over all sequences $\left(l_{1}^{j}, \ldots, l_{t / A}^{j}\right) \in \mathbb{N}^{t / A}$ with $j C_{2} \kappa t<\sum_{i=1}^{t / A} l_{i}^{j} \leq(j+1) C_{2} \kappa t$. Then each summand in (3.13) may, by an application of the Markov property, be rewritten as

$$
\sum_{\left(l_{1}^{j}, \ldots, l_{t / A}^{j}\right)} E_{0}\left(\mathbb{1}\left\{X^{\kappa} \text { has label }\left(l_{1}^{j}, \ldots, l_{t / A-1}^{j}\right)\right\} P_{X^{\kappa}(t-A)}\left(X^{\kappa} \text { has label } l_{t / A}^{j}\right)\right) \text {. }
$$

Note that the number of jumps of a path $\Phi$ that visits $l_{i}^{j} 1$-blocks is at least $\left(l_{i}^{j} / 2^{d}-1\right) A$. This is because for each 1-block there are $\left(2^{d}-1\right)$ 1-blocks with the same time coordinate at $l^{\infty}$-distance one. Hence, we may estimate (3.14) from above by

$$
\sum_{\left(l_{1}^{j}, \ldots, l_{t / A}^{j}\right)} E_{0}\left(\mathbb{1}\left\{X^{\kappa} \text { has label }\left(l_{1}^{j}, \ldots, l_{t / A-1}^{j}\right)\right\}\right) P_{0}\left(N\left(X^{\kappa}, A\right) \geq\left(l_{i}^{j} / 2^{d}-1\right) A\right),
$$

where $N\left(X^{\kappa}, A\right)$ denotes the number of jumps of $X^{\kappa}$ in the time interval [0,A). An iteration of the arguments in (3.14 3.15), together with the tail estimate $P(\operatorname{POISSON}(\lambda) \geq k) \leq$ $e^{-\lambda}(\lambda e)^{k} / k^{k}, k>2 \lambda+1$, for Poisson random variables with mean $\lambda$, yields that for $C^{\prime}>0$ large enough each summand in (3.13) is bounded from above by

$$
\begin{aligned}
& \sum_{\left(l_{1}^{j}, \ldots, l_{t / A}^{j}\right)} \prod_{l_{i}^{j} \geq \kappa C^{\prime}} P_{0}\left(N\left(X^{\kappa}, A\right) \geq\left(l_{i}^{j} / 2^{d}-1\right) A\right) \\
& \quad \leq \sum_{\left(l_{1}^{j}, \ldots, l_{t / A}^{j}\right)} \prod_{l_{i}^{j} \geq \kappa C^{\prime}} e^{-A 2 d \kappa} \exp \left\{-\left(l_{i}^{j} / 2^{d}-2\right) A \log \left(\left[\kappa C^{\prime} / 2^{d}-2\right] / 2 d \kappa e\right)\right\} .
\end{aligned}
$$

(It suffices to pick $C^{\prime}$ such that $\left(C^{\prime} / 2^{d}-2\right) A \geq 4 e A d \kappa+1$, which for $A>1$ and $\kappa>1$ is fulfilled when $C^{\prime} \geq 2^{d}(4 d e+3)$.) Now note that if $C_{2}>2 C^{\prime}$, then for all $j \in \mathbb{N}$,

$$
\sum_{l_{i}^{j} \geq \kappa C^{\prime}} l_{i}^{j} \geq \frac{j t C_{2} \kappa}{2}
$$

Hence, inserting (3.17) into (3.16), choosing $C_{2}$ large enough, and using the fact that for some $a, b \in(0, \infty)$ there are no more than $a e^{b \sqrt{C_{2} \kappa t}}$ such sequences $\left(l_{1}^{j}, \ldots, l_{t / A}^{j}\right)$ (see [7] or [2]), we get that for some $C^{\prime \prime}>0$ the left-hand side of (3.13) is bounded from above by $e^{-C^{\prime \prime} \kappa t}$. Inserting this bound into (3.11), using that $C^{\prime \prime} \rightarrow \infty$ as $C_{2} \rightarrow \infty$, and using (3.12), we get the claim. 


\section{Proof of Proposition 2.2}

Proposition 2.2 is proved in Section 4.2 subject to Propositions 4.14 .2 below, which are stated in Section 4.1 and proved in Sections 56 .

\subsection{Two more propositions}

Endow $\mathbb{Z}$ with the ordering $0 \prec 1 \prec-1 \prec 2 \prec-2 \prec 3 \prec \cdots$. We say that two functions $f, g: \mathbb{Z} \rightarrow \mathbb{R}$ are equimeasurable when

$$
|\{x \in \mathbb{Z}: f(x)>\lambda\}|=|\{x \in \mathbb{Z}: g(x)>\lambda\}| \quad \forall \lambda \geq 0 .
$$

The symmetric decreasing rearrangement of a function $f: \mathbb{Z} \rightarrow \mathbb{R}$ is defined to be the unique non-increasing function $f^{\sharp}: \mathbb{Z} \rightarrow \mathbb{R}$ that is equimeasurable with $f$. Given $A \subseteq \mathbb{Z}, A^{\sharp} \subseteq \mathbb{Z}$ is defined to be the unique set such that $\left(\mathbb{1}_{A}\right)^{\sharp}=\mathbb{1}_{A^{\sharp}}$.

For $B \subseteq \mathbb{Z}^{d} \times[0, t]$, let $\pi_{1}(B) \subseteq \mathbb{Z} \times[0, t]$ be the projection of the spatial part of $B$ onto its first spatial coordinate. Its one-dimensional symmetric decreasing rearrangement is the set

$$
\pi_{1}(B)^{\sharp}=\bigcup_{s \in[0, t]}\left(\left\{x \in \mathbb{Z}:(x, s) \in \pi_{1}(B)\right\}^{\sharp} \times\{s\}\right) .
$$

For $A \geq 1$ and $R \in \mathbb{N}$, an $R$-interval is a time-interval of the form $\left[k A^{R},(k+1) A^{R}\right), 0 \leq$ $k<t / A^{R}$. To make the proof more accessible, we no longer distinguish between badness referring to $\xi \mathbb{1}\{\xi \geq K\}$ and badness referring to $\xi$. Since both potentials satisfy the same mixing assumption (a1) it will be clear from the proof that this does not affect the result.

Proposition 4.1. Let $\Phi \in \Pi\left(k_{*}, t, A\right)$. Then for all $A$ large enough there is a sequence $\left(\delta_{R}\right)_{R \in \mathbb{N}}$ in $(0, \infty)$ satisfying

$$
\sum_{R \in \mathbb{N}} A^{R d} \sqrt{\delta_{R}}<\infty
$$

such that $\xi$-a.s. the number of $R$-intervals in which $\Phi$ crosses more than $\delta_{R} k_{*} /(t / A)$ bad $R$ blocks is bounded from above by $\sqrt{\delta_{R}} t / A^{R}$. A possible choice is $\delta_{R}=K_{1} A^{-8 d^{2} / 3} A^{-4 d(2 d+1) R / 3}$ for some $K_{1}>0$ not depending on $A$ and $R$.

Proposition 4.2. For every $\varepsilon, t>0$, every sequence $\left(B_{R}\right)_{R \in \mathbb{N}}$ in $\mathbb{Z}^{d} \times[0, t]$ and every sequence $\left(C_{R}\right)_{R \in \mathbb{N}}$ in $[0, \infty)$ (see Fig. [3),

$$
E_{0}\left(\exp \left\{\sum_{R=1}^{\varepsilon \log t} C_{R} l_{t}\left(B_{R}\right)\right\}\right) \leq E_{0}\left(\exp \left\{\sum_{R=1}^{\varepsilon \log t} C_{R} l_{t}\left(\pi_{1}\left(B_{R}\right)^{\sharp}\right)\right\}\right) .
$$

\subsection{Proof of Proposition 2.2 subject to two propositions}

Proof. Fix $\varepsilon>0$ and $A \geq 1$ according to Propositions 2.1 and 4.1, and fix $\tilde{\varepsilon}>0$. The proof comes in 6 steps.

1. We begin by introducing some more notation. Define the space-time blocks

$$
B_{R}^{A}(x, k ; \kappa)=\left(\prod_{j=1}^{d}\left[\sqrt{\kappa}(x(j)-1) A^{R}, \sqrt{\kappa}(x(j)+1) A^{R}\right) \cap \mathbb{Z}^{d}\right) \times\left[k A^{R},(k+1) A^{R}\right),
$$



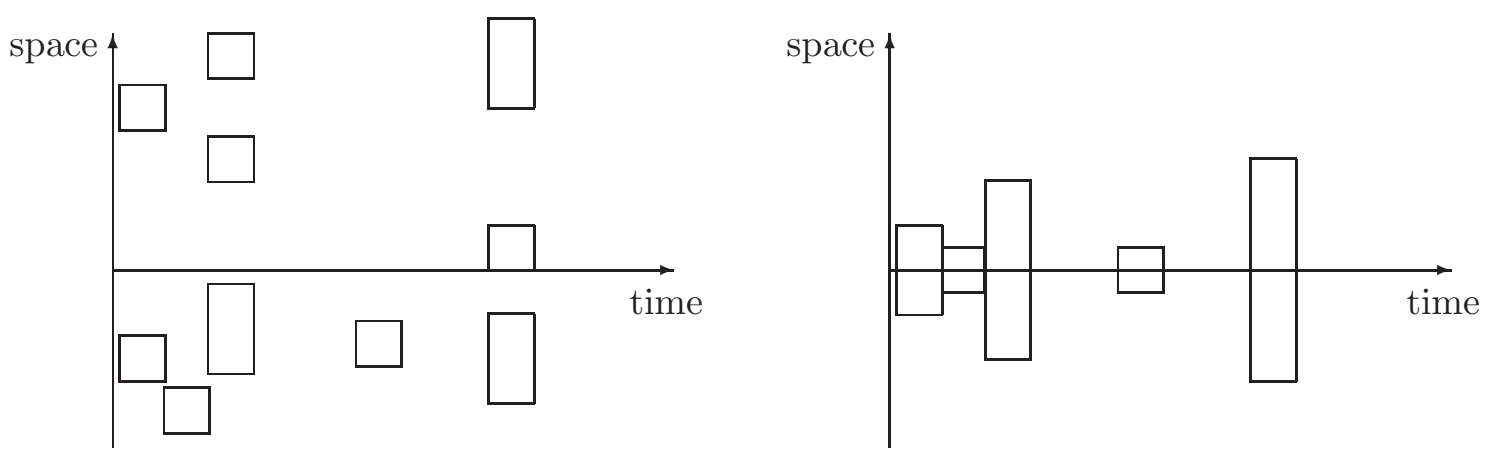

Figure 3: The picture on the left shows a configuration of space-time blocks before its rearrangement, the picture on the right after its rearrangement. Note that in each time-interval the total space volume of the blocks is the same in both configurations.

which we call $(\kappa, R)$-blocks. These blocks are the same as $\tilde{B}_{R}^{A, \alpha}(x, k ; 0,0)=B_{R}^{A, \alpha}(x, k)$ in (1.13) with $\alpha=\sqrt{\kappa}$. Let $k_{*}(\kappa)$ denote the number of $(\kappa, 1)$-blocks that are crossed by $X^{\kappa}$. For $k_{*}(\kappa) \in \mathbb{N}$ and $\left(x_{i}, k_{i}\right)_{0 \leq i<k_{*}(\kappa)} \in\left(\mathbb{Z}^{d} \times \mathbb{N}\right)^{k_{*}(\kappa)}$, write

$$
\bigcup_{\left(x_{i}, k_{i}\right)_{0 \leq i<k_{*}(\kappa)}} B_{1}^{A}\left(x_{i}, k_{i} ; \kappa\right)
$$

to denote the union over all the $(\kappa, 1)$-blocks $B_{1}^{A}\left(x_{i}, k_{i} ; \kappa\right), 0 \leq i<k_{*}(\kappa)$. Likewise, write

$$
\sum_{\left(B_{1}^{A}\left(x_{i}, k_{i} ; \kappa\right)\right)_{0 \leq i<k_{*}(\kappa)}}
$$

to denote the sum over all possible sequences of $(\kappa, 1)$-blocks $B_{1}^{A}\left(x_{i}, k_{i} ; \kappa\right), 0 \leq i<k_{*}(\kappa)$ that can be crossed by a path $\Phi$. Finally, define

$$
\operatorname{BAD}_{R}^{\delta}\left(\left(x_{i}, k_{i}\right)_{0 \leq i<k_{*}(\kappa)}\right)=\left\{B_{R}^{A}(x, k): B_{R}^{A}(x, k) \text { is bad and intersects the union in (4.6) }\right\} .
$$

2. We write $l_{t}\left(\mathrm{BAD}_{R}^{\delta}\right)$ for the local time of $X^{\kappa}$ in $(\delta, b, c)$-bad $R$-blocks up to time $t$, where badness refers both to $\xi \mathbb{1}\{\xi \geq K\}$ and $\xi$. By (2.5), it is enough to show that for all $\kappa$ and $t$ large enough,

$$
E_{0}\left(\exp \left\{4 \sum_{R=1}^{\varepsilon \log t} \delta A^{(R+1) d} l_{t}\left(\mathrm{BAD}_{R}^{\delta}\right)\right\} \mathbb{1}\left\{\exists k_{*} \leq C_{2} \kappa t: X^{\kappa} \in \Pi\left(k_{*}, t, A\right)\right\}\right) \leq e^{\tilde{\varepsilon} t} .
$$

Recall (2.2) to note that the left-hand side of (4.9) equals

$$
\sum_{k_{*}=t / A}^{C_{2} \kappa t} E_{0}\left(\exp \left\{4 \sum_{R=1}^{\varepsilon \log t} \delta A^{(R+1) d} l_{t}\left(\mathrm{BAD}_{R}^{\delta}\right)\right\} \mathbb{1}\left\{X^{\kappa} \text { crosses } k_{*} \text { 1-blocks }\right\}\right) .
$$

To prove (4.9), we attempt to apply Proposition 4.2. To that end, for each $k_{*}$ we must sum over all configurations of $k_{*}$ 1-blocks that may be crossed by $X^{\kappa}$. However, this sum is difficult 
to control, and therefore we do an additional coarse-graining of space-time by considering $(\kappa, R)$-blocks instead of $R$-blocks. To that end we first note that there is a $C_{4}>0$ such that $k_{*}(\kappa) \leq C_{4} k_{*} / \sqrt{\kappa}+2 t / A$ (see Lemma 5.6 in Section 5.4 for a similar statement). To see why, note that if $\Phi$ crosses $l_{i} \leq \sqrt{\kappa}$ 1-blocks in the time-interval $[(i-1) A, i A), 1 \leq i \leq t / A$, then it crosses $l_{i}^{\kappa} \leq 2(\kappa, 1)$-blocks in the same time-interval. Moreover, if $j \sqrt{\kappa}+1 \leq l_{i} \leq(j+1) \sqrt{\kappa}$ for some $j \in \mathbb{N}$, then $l_{i}^{\kappa} \leq j+2 \leq(j+2) l_{i} / j \sqrt{\kappa}$. Hence, the total number of $(\kappa, 1)$-blocks that may be crossed by $\Phi$ is bounded from above by

$$
\sum_{i=1}^{t / A} l_{i}^{\kappa} \leq \sum_{\substack{1 \leq i \leq t / A \\ l_{i} \leq \sqrt{\kappa}}} 2+\sum_{\substack{1 \leq i \leq t / A \\ l_{i} \geq \sqrt{\kappa}+1}} \frac{3 l_{i}}{\sqrt{\kappa}} \leq 2 t / A+\frac{3 k_{*}}{\sqrt{\kappa}}
$$

Thus, (4.10) is bounded from above by

$$
\sum_{k_{*}(\kappa)=t / A}^{C_{2} C_{4} \sqrt{\kappa} t+2 t / A} E_{0}\left(\exp \left\{4 \sum_{R=1}^{\varepsilon \log t} \delta A^{(R+1) d} l_{t}\left(\mathrm{BAD}_{R}^{\delta}\right)\right\} \mathbb{1}\left\{X^{\kappa} \text { crosses } k_{*}(\kappa)(\kappa, 1) \text {-blocks }\right\}\right) .
$$

To analyze (4.12), fix $k_{*}(\kappa) \in\left[t / A, C_{2} C_{4} \sqrt{\kappa} t+2 t / A\right]$. Summing over all possible ways to cross $k_{*}(\kappa)(\kappa, 1)$-blocks and recalling (4.8), we may estimate each summand in (4.12) by

$$
\begin{aligned}
\sum_{\left(B_{1}^{A}\left(x_{i}, k_{i} ; \kappa\right)\right)_{0 \leq i<k_{*}(\kappa)}} E_{0}(\exp & \left\{4 \sum_{R=1}^{\varepsilon \log t} \delta A^{(R+1) d} l_{t}\left(\operatorname{BAD}_{R}^{\delta}\left(\left(x_{i}, k_{i}\right)_{0 \leq i<k_{*}(\kappa)}\right)\right)\right\} \\
& \left.\times \mathbb{1}\left\{X^{\kappa} \operatorname{crosses} B_{1}^{A}\left(x_{i}, k_{i} ; \kappa\right), 0 \leq i<k_{*}(\kappa)\right\}\right) .
\end{aligned}
$$

By Cauchy-Schwarz, (4.13) is at most

$$
\begin{gathered}
\sum_{\left(B_{1}^{A}\left(x_{i}, k_{i} ; \kappa\right)\right)_{0 \leq i<k_{*}(\kappa)}}\left[E_{0}\left(\exp \left\{8 \sum_{R=1}^{\varepsilon \log t} \delta A^{(R+1) d} l_{t}\left(\operatorname{BAD}_{R}^{\delta}\left(\left(x_{i}, k_{i}\right)_{0 \leq i<k_{*}(\kappa)}\right)\right)\right\}\right)\right]^{1 / 2} \\
\times\left[P_{0}\left(X^{\kappa} \operatorname{crosses} B_{1}^{A}\left(x_{i}, k_{i}, \kappa\right), 0 \leq i<k_{*}(\kappa)\right)\right]^{1 / 2} .
\end{gathered}
$$

3. By Proposition 4.2, the first factor in the summand of (4.14) is not more than

$$
\left[E_{0}\left(\exp \left\{8 \sum_{R=1}^{\varepsilon \log t} \delta A^{(R+1) d} l_{t}\left(\pi_{1}\left(\operatorname{BAD}_{R}^{\delta}\left(\left(x_{i}, k_{i}\right)_{0 \leq i<k_{*}(\kappa)}\right)\right)^{\sharp}\right)\right\}\right)\right]^{1 / 2} .
$$

Next, if $X^{\kappa}$ crosses $k_{*}(\kappa)(\kappa, 1)$-blocks $B_{1}^{A}(x, k ; \kappa)$, then a trivial counting estimate yields that $X^{\kappa}$ crosses at most $k_{*}(\kappa) \sqrt{\kappa} 1$-blocks. Therefore, by Proposition 4.1, the number of $R$ intervals in which $X^{\kappa}$ crosses more than $\delta_{R} k_{*}(\kappa) \sqrt{\kappa} A / t$ bad $R$-blocks is bounded from above by $\sqrt{\delta_{R}} t / A^{R}$. We call these $R$-intervals $R$-atypical. Similarly, an $R$-interval is called $R$-typical, if the number of bad $R$-blocks crossed by $X^{\kappa}$ is bounded by $\delta_{R} k_{*}(\kappa) \sqrt{\kappa} A / t$. Define

$$
R^{*}\left(k_{*}(\kappa)\right)=\max \left\{R \in \mathbb{N}: \delta_{R} k_{*}(\kappa) \sqrt{\kappa} A / t \geq 1\right\} .
$$




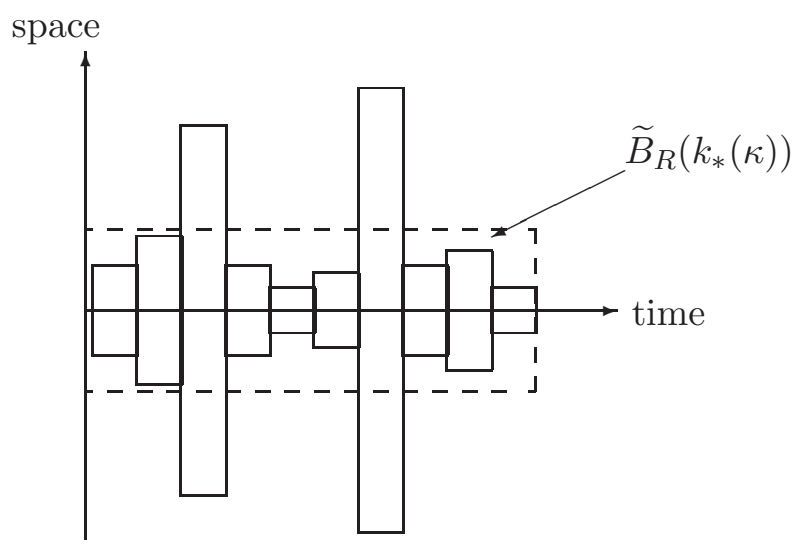

Figure 4: The picture shows a possible configuration of bad $R$-blocks after its rearrangement. There are two time-intervals in which the number of bad $R$-blocks is atypically large, i.e., larger than $\delta_{R} k_{*}(\kappa) \sqrt{\kappa} A / t$. The local time in these bad $R$-blocks can be bounded from above by the total length of these time-intervals, which is at most $\sqrt{\delta_{R}} t$. The local time of the bad $R$-blocks in the other timeintervals can be bounded from above by the local time of the enveloping dashed block, i.e., $\widetilde{B}_{R}\left(k_{*}(\kappa)\right)$.

If $R>R^{*}\left(k_{*}(\kappa)\right)$, then there are no bad $R$-blocks in $R$-typical intervals. (By the choice of $R$, their number is strictly less than one and therefore is zero.) Hence the local time in bad $R$-blocks is determined by the local time in bad $R$-blocks, which lie in $R$-atypical intervals. Consequently,

$$
l_{t}\left(\pi_{1}\left(\operatorname{BAD}_{R}^{\delta}\left(\left(x_{i}, k_{i}\right)_{0 \leq i<k_{*}(\kappa)}\right)\right)^{\sharp}\right) \leq\left(\sqrt{\delta_{R}} t / A^{R}\right) A^{R}=\sqrt{\delta_{R}} t .
$$

On the other hand, if $1 \leq R \leq R^{*}\left(k_{*}(\kappa)\right)$ (see Fig. 4), then there is a contribution coming from $R$-typical intervals as well, and so

$$
l_{t}\left(\pi_{1}\left(\operatorname{BAD}_{R}^{\delta}\left(\left(x_{i}, k_{i}\right)_{0 \leq i<k_{*}(\kappa)}\right)\right)^{\sharp}\right) \leq \sqrt{\delta_{R}} t+l_{t}\left(\widetilde{B}_{R}\left(k_{*}(\kappa)\right)\right),
$$

where

$$
\widetilde{B}_{R}\left(k_{*}(\kappa)\right)=\left(\left[-\frac{1}{2} A^{R} \delta_{R} k_{*}(\kappa) \sqrt{\kappa} A / t, \frac{1}{2} A^{R} \delta_{R} k_{*}(\kappa) \sqrt{\kappa} A / t\right) \cap \mathbb{Z}\right) \times[0, t] .
$$

Hence, 4.15) is bounded from above by

$$
\left[E_{0}\left(\exp \left\{8 \sum_{R=1}^{R^{*}\left(k_{*}(\kappa)\right)} \delta A^{(R+1) d} l_{t}\left(\widetilde{B}_{R}\left(k_{*}(\kappa)\right)\right)\right\}\right)\right]^{1 / 2} \exp \left\{4 \sum_{R \in \mathbb{N}} \delta A^{(R+1) d} \sqrt{\delta_{R}} t\right\} .
$$

For $A$ large enough, by Proposition 4.1 and the specific choice of $\left(\delta_{R}\right)_{R \in \mathbb{N}}$ in Propostion 4.1 , the sum in the second term is $\leq \tilde{\varepsilon} t / 2$.

4. To estimate the first factor in (4.20) and control the second factor in the summand of (4.14), we need the following two lemmas whose proof is deferred to Appendix A.

Lemma 4.3. Let $X^{\kappa}$ be simple random walk on $\mathbb{Z}$ with step rate $2 \kappa$. There is a $K_{2}>0$ such that for all $\kappa>0$, all $n \in \mathbb{N}$, all $\beta_{1}, \beta_{2}, \ldots, \beta_{n} \geq 0$ and all nested finite intervals $\emptyset=I_{0} \subseteq I_{1} \subseteq I_{2} \subseteq \cdots \subseteq I_{n} \subseteq \mathbb{Z}$

$$
\log E_{0}\left(\exp \left\{\sum_{i=1}^{n} \beta_{i} \sum_{x \in I_{i}} l_{t}\left(X^{\kappa}, x\right)\right\}\right) \leq \frac{K_{2} t}{\sqrt{\kappa}} \sum_{i=1}^{n}\left[\left|I_{i} \backslash I_{i-1}\right|\left(\sum_{j=i}^{n} \beta_{j}\right)^{3 / 2}\right]+o(t),
$$


where $l_{t}\left(X^{\kappa}, x\right)$ is the local time of $X^{\kappa}$ at site $x$ up to time $t$.

Lemma 4.4. There are $C_{5}, C_{6}>0$ such that for all $\kappa, t>0$ large enough, all $A>0$ and all $k_{*}(\kappa) \geq C_{5} t$,

$$
P_{0}\left(X^{\kappa} \text { crosses } k_{*}(\kappa)(\kappa, 1) \text {-blocks }\right) \leq e^{-C_{6} A k_{*}(\kappa)} .
$$

Note that $A^{R+1} \delta_{R+1}<A^{R} \delta_{R}$, and so $\widetilde{B}_{R+1}\left(k_{*}(\kappa)\right) \subseteq \widetilde{B}_{R}\left(k_{*}(\kappa)\right)$ for all $R \in \mathbb{N}$. Moreover, for $k_{*}(\kappa) \leq C_{2} C_{4} \sqrt{\kappa} t+2 t / A$ and $1 \leq R \leq R^{*}\left(k_{*}(\kappa)\right)$ we have that the cardinality of the spatial part of the blocks defined in (4.19) satisfies $\left|\widetilde{B}_{R}\left(k_{*}(\kappa)\right)\right| \leq\left|\widetilde{B}_{1}\left(k_{*}(\kappa)\right)\right| \leq A^{2} \delta_{1} C_{2} C_{4} \kappa+2 A \delta_{1} \sqrt{\kappa}$, which is bounded uniformly in $t$. To apply Lemma 4.3, we choose $t_{0}$ (which may depend on $\kappa)$ such that for each family of intervals $I_{1}, \ldots, I_{R^{*}\left(k_{*}\right)}, k_{*}(\kappa) \in\left[t / A, C_{2} C_{4} \sqrt{\kappa} t+2 t / A\right]$, with the property that $\left|I_{i}\right| \in\left[A, A^{2} \delta_{1} C_{2} C_{4} \kappa+2 A \delta_{1} \sqrt{\kappa}\right.$ for all $i \in\left\{1, \ldots, R^{*}\left(k_{*}(\kappa)\right)\right\}$ the assertion of Lemma 4.3 holds uniformly in $t \geq t_{0}$. Then, for all $t \geq t_{0}$, the expectation in the left-hand side of (4.20) is at most

$$
\exp \left\{\frac{K_{2} t}{\sqrt{\kappa}} \sum_{R=1}^{R^{*}\left(k_{*}(\kappa)\right)}\left[\left|\widetilde{B}_{R}\left(k_{*}(\kappa)\right) \backslash \widetilde{B}_{R+1}\left(k_{*}(\kappa)\right)\right|\left(\sum_{j=1}^{R} 8 \delta A^{(j+1) d}\right)^{3 / 2}\right]+o(t)\right\},
$$

where $\widetilde{B}_{R^{*}\left(k_{*}(\kappa)\right)+1}\left(k_{*}(\kappa)\right)=\emptyset$. Next, note that

$$
\left|\widetilde{B}_{R}\left(k_{*}(\kappa)\right) \backslash \widetilde{B}_{R+1}\left(k_{*}(\kappa)\right)\right| \leq \frac{A^{R} \delta_{R} k_{*}(\kappa) \sqrt{\kappa} A}{t} .
$$

Therefore the first term in the exponent of (4.23), may be estimated from above by

$$
\begin{aligned}
\frac{K_{2} t}{\sqrt{\kappa}} \sum_{R=1}^{R^{*}\left(k_{*}(\kappa)\right)} & {\left[\frac{A^{R} \delta_{R} k_{*}(\kappa) \sqrt{\kappa} A}{t}\left(\sum_{j=1}^{R} 8 \delta A^{(j+1) d}\right)^{3 / 2}\right] } \\
& \leq(8 \delta)^{3 / 2} K_{2} k_{*}(\kappa) A^{3 d / 2+1} \sum_{R=1}^{R^{*}\left(k_{*}(\kappa)\right)}\left[A^{R} \delta_{R}\left(\sum_{j=1}^{R} A^{j d}\right)^{3 / 2}\right] .
\end{aligned}
$$

Furthermore,

$$
\sum_{j=1}^{R} A^{j d}=\frac{A^{d}}{A^{d}-1}\left(A^{R d}-1\right) \leq C A^{R d},
$$

where $C>0$ does not depend on $A$. Hence, the right-hand side of (4.25) is at most

$$
(8 \delta)^{3 / 2} C K_{2} k_{*}(\kappa) A^{3 d / 2+1} \sum_{R=1}^{R^{*}\left(k_{*}(\kappa)\right)} A^{R} \delta_{R} A^{3 R d / 2} .
$$

Recalling our choice of $\delta_{R}$ in Proposition 4.1, we can estimate the sum in (4.27) from above by

$$
K_{1} A^{-8 d^{2} / 2} A^{-D(d)}\left[\frac{1-A^{-R^{*}\left(k_{*}(\kappa)\right) D(d)}}{1-A^{-D(d)}}\right] .
$$

with $D(d)=\left(16 d^{2}-d-6\right) / 6>0$. Since $A>3$ by Proposition 2.1, the last term in (4.28) is bounded uniformly in $A$ and $R^{*}\left(k_{*}(\kappa)\right)$. Inserting (4.28) into (4.27), we see that there is a $C_{7}>0$, not depending on $A$, such that the exponent in (4.23) is bounded from above by 
$(8 \delta)^{3 / 2} C_{7} A^{-D^{\prime}(d)} k_{*}(\kappa)+o(t)$ with $D^{\prime}(d)=\left(16 d^{2}-5 d-6\right) / 3>0$, where $o(t)$ is uniform in $t \geq t_{0}$ for all $k_{*}(\kappa) \in\left[t / A, C_{2} C_{4} \sqrt{\kappa} t+2 t / A\right]$.

5. It remains to estimate (recall (4.14))

$$
\sum_{\left(B_{1}^{A}\left(x_{i}, k_{i} ; \kappa\right)\right)_{0 \leq i<k_{*}(\kappa)}}\left[P_{0}\left(X^{\kappa} \operatorname{crosses} B_{1}^{A}\left(x_{i}, k_{i}, \kappa\right), 0 \leq i<k_{*}(\kappa)\right)\right]^{1 / 2} .
$$

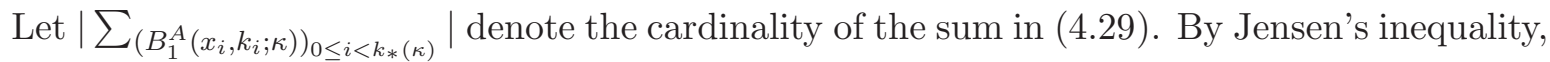
(4.29) is not more than (recall (4.7))

$$
\begin{aligned}
& \left|\sum_{\left(B_{1}^{A}\left(x_{i}, k_{i} ; \kappa\right)\right)_{0 \leq i<k_{*}(\kappa)}}\right|^{1 / 2}\left[\sum_{\left(B_{1}^{A}\left(x_{i}, k_{i} ; \kappa\right)\right)_{0 \leq i<k_{*}(\kappa)}} P_{0}\left(X^{\kappa} \operatorname{crosses} B_{1}^{A}\left(x_{i}, k_{i} ; \kappa\right), 0 \leq i<k_{*}(\kappa)\right)\right]^{1 / 2} \\
& =\left|\sum_{\left(B_{1}^{A}\left(x_{i}, k_{i} ; \kappa\right)\right)_{0 \leq i<k *(\kappa)}}\right|^{1 / 2}\left[P_{0}\left(X^{\kappa} \operatorname{crosses} k_{*}(\kappa)(\kappa, 1) \text {-blocks }\right)\right]^{1 / 2} .
\end{aligned}
$$

To estimate the first term in the right-hand side of (4.30), note that $\left|\sum_{\left(B_{1}^{A}\left(x_{i}, k_{i} ; \kappa\right)\right)_{0 \leq i<k *}(\kappa)}\right|$ equals the number of different ways to visit $k_{*}(\kappa)(\kappa, 1)$-blocks. Hence, there is a $C_{8}>0$ such that $\left|\sum_{\left(B_{1}^{A}\left(x_{i}, k_{i} ; \kappa\right)\right)_{0 \leq i<k_{*}(\kappa)}}\right|$ is bounded from above by $e^{C_{8} k_{*}(\kappa)}$ (see also Lemma [5.5] in Section 5.4). Therefore, by Lemma 4.4, for $k_{*}(\kappa) \geq C_{5} t$ and $\kappa$ large enough, the right-hand side of (4.30) may be estimated from above by

$$
e^{C_{8} k_{*}(\kappa)} e^{-C_{6} A k_{*}(\kappa)}
$$

6. We are now in a position to complete the proof of (4.9). Combining the estimates in (4.15), (4.20) and (4.254.4.31), we get for $t \geq t_{0}$ (see the lines following (4.28)),

$$
\begin{aligned}
& E_{0}\left(\exp \left\{4 \sum_{R=1}^{\varepsilon \log t} \delta A^{(R+1) d} l_{t}\left(\mathrm{BAD}_{R}\right)\right\} \mathbb{1}\left\{\exists k_{*} \leq C_{2} \kappa t: X^{\kappa} \in \Pi\left(k_{*}, t, A\right)\right\}\right) \\
& \leq e^{\tilde{\varepsilon} t / 2} \sum_{k_{*}(\kappa)=t / A}^{C_{5} t-1} e^{(8 \delta)^{3 / 2} C_{7} A^{-D^{\prime}(d)} k_{*}(\kappa)+o(t)} \\
& \quad+e^{\tilde{\varepsilon} t / 2} \sum_{k_{*}(\kappa)=C_{5} t}^{C_{2} C_{4} \sqrt{\kappa} t+2 t / A} e^{(8 \delta)^{3 / 2} C_{7} A^{-D^{\prime}(d)} k_{*}(\kappa)+o(t)} e^{C_{8} k_{*}(\kappa)} e^{-C_{6} A k_{*}(\kappa)} \\
& \leq e^{\tilde{\varepsilon} t / 2} C_{5} t e^{(8 \delta)^{3 / 2} C_{7} C_{5} A^{-D^{\prime}(d)} t+o(t)}+e^{\tilde{\varepsilon} t / 2} C_{9} \\
& \leq e^{2 \tilde{\varepsilon} t}
\end{aligned}
$$

where we use that the sum in the third line of (4.32) is finite for $A$ large enough (which requires that $\varepsilon$ is small enough; recall Proposition 2.1). This settles (4.9) and completes the proof of Proposition 2.2. 


\section{$5 \quad$ Proof of Proposition 4.1}

The proof is given in Section 5.1 subject to Lemma 5.1 below. This lemma is stated in Section 5.1 and proved in Sections 5.2 5.5. Recall the definition of $\Xi_{R}^{A, k_{*}}$ in (3.1). Throughout this section we abbreviate

$$
\widetilde{\delta}_{R}=A^{-2 d(2 d+1) R} .
$$

\subsection{Proof of Proposition 4.1 subject to a further lemma}

Lemma 5.1. There is a $C>0$ such that $\xi$-a.s. for all $A$ and $m$ large enough, all $R \in \mathbb{N}$ and all $k_{*} \in \mathbb{N}$,

$$
\Xi_{R}^{A, k_{*}} \leq C A^{-\left(4 d^{2}-1\right)} A^{-R} \widetilde{\delta}_{R} k_{*} .
$$

We are now ready to prove Proposition 4.1

Proof. Let $\Phi \in \Pi\left(k_{*}, t, A\right)$ and $R \in \mathbb{N}$. Suppose that there is a $\delta_{R}>0$ such that there are at least $\sqrt{\delta_{R}} t / A^{R} R$-intervals in which $\Phi$ crosses more than $\delta_{R} k_{*} /(t / A)$ bad $R$-blocks. In all of these $R$-intervals $\Phi$ crosses at least

$$
\frac{\sqrt{\delta_{R}} t}{A^{R}} \frac{\delta_{R} k_{*}}{(t / A)}=\delta_{R}^{3 / 2} A^{-(R-1)} k_{*}
$$

bad $R$-blocks. Lemma 5.1 implies that $\xi$-a.s. $\delta_{R}^{3 / 2} A^{-(R-1)} \leq C A^{-\left(4 d^{2}-1\right)} A^{-R} \widetilde{\delta}_{R}$, which is the same as $\delta_{R}^{3 / 2} \leq C A^{-4 d^{2}} A^{-2 d(2 d+1) R}$. This yields the claim below (4.3) with $K_{1}=C^{2 / 3}$.

\subsection{Proof of Lemma 5.1 subject to two further lemmas}

The proof of Lemma 5.1 is a modification of the proof of [3, Lemma 3.5] and is based on Lemmas 5.25 .3 below, which count bad $R$-blocks. The proof of the second lemma is deferred to Section 5.3 .

For $A \geq 1, R \in \mathbb{N}$ and $\Phi \in \Pi\left(k_{*}, t, A\right)$, define

$\Psi_{R}^{A}(\Phi)=$ number of good $(R+1)$-blocks crossed by $\Phi$ containing a bad $R$-block,

$\Psi_{R}^{A, k_{*}}=\sup _{\Phi \in \Pi\left(k_{*}, t, A\right)} \Psi_{R}^{A}(\Phi)$.

Lemma 5.2. There is a $C^{\prime}>0$ such that for all $A$ and $m$ large enough

$$
\mathbb{P}\left(\Psi_{R}^{A, k_{*}} \geq C^{\prime} A^{-R} \widetilde{\delta}_{R} k_{*} \text { for some } R \in \mathbb{N} \text { and some } k_{*} \in \mathbb{N}_{0}\right)
$$

is summable over $t \in \mathbb{N}$. A possible choice is $C^{\prime}=3$.

Lemma 5.3. For all $\varepsilon>0$ there is an $A=A(\varepsilon)>3$ such that $\xi$-a.s. there is a $t_{0}>0$ such that for all $R \in \mathbb{N}$, all $k_{*} \in \mathbb{N}$ and all $t \geq t_{0}$,

$$
\Xi_{R}^{A, k_{*}} \leq A^{d+1} \sum_{i=1}^{\varepsilon \log t-R-1} 2^{d i} A^{(d+1) i} \Psi_{R+i}^{A, k_{*}} .
$$


Proof. Lemma 5.3 is the same as [3, Lemma 3.7]. The idea is to look at a bad $R$-block and check whether it is contained in a good $(R+1)$-block or in a bad $(R+1)$-block. An iteration over $R$, combined with a simple counting argument and Lemma 3.1, yields the claim.

We are now ready to prove Lemma 5.1.

Proof. By Lemma 5.2, $\xi$-a.s. for $t$ large enough $\Psi_{R}^{A, k_{*}} \leq C^{\prime} A^{-R} \widetilde{\delta}_{R} k_{*}$ for all $R \in \mathbb{N}$ and all $k_{*} \in \mathbb{N}$. By Lemma 5.3. recalling that $\widetilde{\delta}_{R}=A^{-2 d(2 d+1) R}$, we may estimate

$$
\begin{aligned}
\Xi_{R}^{A, k_{*}} & \leq A^{d+1} \sum_{i \geq 1} 2^{d i} A^{(d+1) i} C^{\prime} A^{-(R+i)} \widetilde{\delta}_{R+i} k_{*} \\
& =C^{\prime} A^{d+1} A^{-R} \widetilde{\delta}_{R} k_{*} \sum_{i \geq 1} 2^{d i} A^{(d+1) i} A^{-i} A^{-2 d(2 d+1) i} \\
& =C^{\prime} A^{d+1} A^{-R} \widetilde{\delta}_{R} k_{*} \frac{2^{d} A^{d} A^{-2 d(2 d+1)}}{1-2^{d} A^{d} A^{-2 d(2 d+1)}} .
\end{aligned}
$$

Note that for $A \geq A_{0}>1$ there is a $C>0$, depending on $A_{0}$ but not on $A$, such that the term in the right-hand side of (5.7) is bounded from above by

$$
C A^{-\left(4 d^{2}-1\right)} A^{-R} \widetilde{\delta}_{R} k_{*},
$$

which yields the claim.

\subsection{Proof of Lemma 5.2 subject to a further lemma}

The proof of Lemma 5.2 is based on Lemma 5.4 below. Let $x \in \mathbb{Z}^{d}$ and $k, R \in \mathbb{N}$. Abbreviate

$$
\chi^{A}(x, k)=\mathbb{1}\left\{B_{R+1}^{A}(x, k) \text { is good but contains a bad } R \text {-block }\right\} .
$$

Lemma 5.4. There is a $C>0$ such that for all $A$ and $m$ large enough, all $R \in \mathbb{N}$ and all $k_{*} \in \mathbb{N}$,

$$
\mathbb{P}\left(\begin{array}{c}
\text { there is a path that crosses } k_{*} 1 \text {-blocks and intersects } \\
\text { at least } 3 A^{-R} \widetilde{\delta}_{R} k_{*} \text { blocks } B_{R+1}^{A}(x, k) \text { with } \chi^{A}(x, k)=1
\end{array}\right) \leq \exp \left\{-C A^{-R} \widetilde{\delta}_{R} k_{*}\right\} .
$$

We are now ready to prove Lemma 5.2 .

Proof. First note that $k_{*} \geq t / A$ and that, $\xi$-a.s. for $t$ large enough, $1 \leq R \leq \varepsilon \log t$, by Lemma 3.1. For each such $R$, we have by Lemma 5.4 .

$$
\begin{aligned}
& \mathbb{P}\left(\begin{array}{l}
\text { there is a path that crosses } k_{*} 1 \text {-blocks and intersects at least } \\
3 A^{-R} \widetilde{\delta}_{R} k_{*} \text { blocks } B_{R+1}^{A}(x, k) \text { with } \chi^{A}(x, k)=1 \text { for some } k_{*} \geq t / A
\end{array}\right) \\
& \leq \sum_{k_{*} \geq t / A} \exp \left\{-C A^{-R} \widetilde{\delta}_{R} k_{*}\right\} \leq \frac{\exp \left\{-C A^{-R} \widetilde{\delta}_{R} t / A\right\}}{1-\exp \left\{-C A^{-R} \widetilde{\delta}_{R}\right\}} .
\end{aligned}
$$

Because $1 \leq R \leq \varepsilon \log t$ and $R \mapsto A^{-R} \widetilde{\delta}_{R}$ is non-increasing, the numerator in the right-hand side of (5.11) is bounded from above by $\exp \left\{-C A^{-\varepsilon \log t} \widetilde{\delta}_{\varepsilon} \log t t / A\right\}$ while the denominator is 
bounded from below by $1-\exp \left\{-C A^{-\varepsilon \log t} \widetilde{\delta}_{\varepsilon \log t}\right\}$. Using the choice of $A$ in Lemma 3.1. we see that (5.11) is bounded from above by

$$
\frac{\exp \left\{-C t^{1-a^{-1}} / A\right\}}{1-\exp \left\{-C t^{-a^{-1}}\right\}}, \quad a>1 .
$$

Note that this is of order $\exp \left\{-C^{\prime} t^{\tilde{\varepsilon}}\right\}$ for some $C^{\prime}, \tilde{\varepsilon}>0$, and so the probability in (5.5) is bounded from above by $(\varepsilon \log t) \exp \left\{-C^{\prime} t^{\tilde{\varepsilon}}\right\}$, which is summable over $t \in \mathbb{N}$.

\subsection{Proof of Lemma 5.4 subject to two further lemmas}

The proof of Lemma 5.4 is based on Lemmas 5.555 .6 below, which are proved in Section 5.5 .

Proof. Our first further lemma reads:

Lemma 5.5. There is a $C>0$ such that for all $l \in \mathbb{N}$ and $R \in \mathbb{N}$ there are no more than $e^{C l}$ possible ways for $\Phi$ to visit at most $l$ R-blocks.

Fix $R \in \mathbb{N}$. We divide blocks into equivalence classes such that blocks belonging to the same equivalence class can essentially be treated as independent. To that end, we take $a_{1}, a_{2} \in \mathbb{N}$ according to condition (a1) in Definition 1.5 and say that $(x, k)$ and $\left(x^{\prime}, k^{\prime}\right)$ are equivalent when

$$
x=x^{\prime}\left(\bmod a_{1}\right), \quad k=k^{\prime}\left(\bmod a_{2}\right) .
$$

We denote the set of corresponding representants by $([x],[k])$, and write $\sum_{([x],[k])}$ to denote the sum over all equivalence classes. Note that the left-hand side of (5.10) is bounded from above by

$$
\sum_{([x],[k])} \mathbb{P}\left(\begin{array}{l}
\text { there is a path that crosses } k_{*} 1 \text {-blocks and intersects } \\
\text { at least } 3 A^{-R} \widetilde{\delta}_{R} k_{*} / a_{1}^{d} a_{2} \text { blocks } B_{R+1}^{A}(x, k) \\
\text { with } \chi^{A}(x, k)=1 \text { and }(x, k) \equiv([x],[k])
\end{array}\right) .
$$

Fix an equivalence class. Put $\rho_{R}=A^{-4 d(2 d+1)(d+1) R}$ (recall (1.17)). To control the cardinality of the number of different ways to visit a given number of $(R+1)$-blocks, we consider enlarged blocks, namely, we let

$$
L=L(R)=\left(1 / \rho_{R}\right)^{1 /(d+1)}
$$

and define

$$
\tilde{B}_{R}^{A}(x, k)=\left(\prod_{j=1}^{d}\left[L x(j) A^{R}, L(x(j)+1) A^{R}\right) \cap \mathbb{Z}^{d}\right) \times\left[L k A^{R}, L(k+1) A^{R}\right) .
$$

Our second further lemma reads:

Lemma 5.6. If $\Phi$ crosses $k_{*} 1$-blocks, then for all $R \in \mathbb{N}$ it crosses no more than $l_{R}=$ $3 k_{*} / A^{R-1} L$ blocks $\widetilde{B}_{R}^{A}(x, k)$.

We write

$$
\bigcup_{\left(x_{i}, k_{i}\right)_{0 \leq i<l} R+1} \tilde{B}_{R+1}^{A}\left(x_{i}, k_{i}\right)
$$


to denote the union over at most $l_{R+1}$ blocks $\widetilde{B}_{R}^{A}(x, k)$ and

$$
\sum_{\left(\tilde{B}_{R+1}^{A}\left(x_{i}, k_{i}\right)\right)_{0 \leq i<l_{R+1}}}
$$

to denote the sum over all possible sequences of at most $l_{R+1}$ blocks $\tilde{B}_{R+1}^{A}\left(x_{i}, k_{i}\right)$ that can be crossed by a path $\Phi$. Since each block $B_{R+1}^{A}(x, k)$ that may be crossed by $\Phi$ lies in the union of (5.17), we may estimate the probability under the sum in (5.14) from above by

$$
\sum_{\left(\tilde{B}_{R+1}^{A}\left(x_{i}, k_{i}\right)\right)_{0 \leq i<l_{R+1}}} \mathbb{P}\left(\begin{array}{l}
\text { the union in }(5.17) \text { contains at least } 3 A^{-R} \widetilde{\delta}_{R} k_{*} / a_{1}^{d} a_{2} \text { blocks } \\
B_{R+1}^{A}(x, k) \text { with } \chi^{A}(x, k)=1 \text { and }(x, k) \equiv([x],[k])
\end{array}\right) .
$$

Next, note that the union in (5.17) contains at most $l_{R+1} L^{d+1}(R+1)$-blocks and that there are $\left(\begin{array}{c}l_{R+1} L^{d+1} \\ n\end{array}\right)$ ways of choosing $n$ blocks $B_{R+1}^{A}(x, k)$ with $\chi^{A}(x, k)=1$ from $l_{R+1} L^{d+1}$ $(R+1)$-blocks. Hence, by the mixing condition in (1.17) for $A$ and $m$ large enough, each summand in (5.19) is bounded from above by

$$
\sum_{n=\delta_{R} k_{R+1} / a_{1}^{d} a_{2}}^{l_{R+1} L^{d+1}}\left(\begin{array}{c}
l_{R+1} L^{d+1} \\
n
\end{array}\right)\left(\rho_{R}\right)^{n} \leq\left(1-\rho_{R}\right)^{-l_{R+1} L^{d+1}} \mathbb{P}\left(T \geq 3 A^{-R} \widetilde{\delta}_{R} k_{*} / a_{1}^{d} a_{2}\right),
$$

where $T=\operatorname{BINOMIAL}\left(l_{R+1} L^{d+1}, \rho_{R}\right)$. Since

$$
\mathbb{E}(T)=\rho_{R} l_{R+1} L^{d+1}=l_{R+1}=3 k_{*} / A^{R} L=3 A^{-R} A^{-4 d(2 d+1) R} k_{*}=3 A^{-R} \widetilde{\delta}_{R}^{2} k_{*} \ll 3 A^{-R} \widetilde{\delta}_{R} k_{*},
$$

we can apply standard large deviation estimates to bound the right-hand side of (5.20). Indeed, by Bernstein's inequality, there is a $C^{\prime}>0$ (depending on $a_{1}$ and $a_{2}$ only) such that for all $A$ and $m$ large enough,

$$
\mathbb{P}\left(T \geq 3 A^{-R} \widetilde{\delta}_{R} k_{*} / a_{1}^{d} a_{2}\right) \leq e^{-C^{\prime} 3 A^{-R} \widetilde{\delta}_{R} k_{*} / a_{1}^{d} a_{2}} .
$$

Moreover, there is a $C^{\prime \prime}>0$ (not depending on $A$, provided $A$ is large enough) such that

$$
\left(1-\rho_{R}\right)^{-l_{R+1} L^{d+1}} \leq e^{\rho_{R} l_{R+1} L^{d+1} /\left(1-\rho_{R}\right)} \leq e^{C^{\prime \prime} 3 A^{-R} \widetilde{\delta}_{R}^{2} k_{*}} .
$$

Furthermore, by Lemma 5.5, and after a possible increase of $C^{\prime \prime}$, the sum in (5.18) contains at most $e^{C^{\prime \prime} l_{R+1}}=e^{C^{\prime \prime} 3 A^{-R} \widetilde{\delta}_{R}^{2} k_{*}}$ elements. Hence, combining (5.14), (5.19 5.20) and (5.22 5.23), we see that the left-hand side of (5.10) is bounded from above by $e^{-C A^{-R} \widetilde{\delta}_{R} k_{*}}$, with $C$ such that $C A^{-R} \widetilde{\delta}_{R} k_{*} \geq\left(C^{\prime} / a_{1}^{d} a_{2}-\widetilde{\delta}_{R} 2 C^{\prime \prime}\right) 3 A^{-R} \widetilde{\delta}_{R} k_{*}$, which yields the claim in (5.10).

\subsection{Proof of Lemmas $5.5 \square .6$}

Proof. For the proof of Lemma 5.5, see the proof of [3, Claim 3.8]. The proof of Lemma 5.6 goes as follows. Let $R \in \mathbb{N}$. Divide time into intervals of length $L A^{R}$. Let $l_{i}^{L}$ and $l_{i}$ be the number of blocks $\widetilde{B}_{R}^{A}(x, k)$, respectively, 1-blocks, crossed by $X^{\kappa}$ in the $i$-th time interval $\left[(i-1) L A^{R}, i L A^{R}\right), 1 \leqq i \leq t / L A^{R}$. Note that $l_{i} \geq L A^{R-1}$ because the length of the timeinterval of each block $\widetilde{B}_{R}^{A}(x, k)$ is $L A^{R}$, which may be divided into $L A^{R-1}$ time-intervals of 
length $A$. Moreover $X^{\kappa}$ has to cross at least one 1-block in each such interval of length $A$. Also note that if $l_{i}=L A^{R-1}$, then $l_{i}^{L} \leq 2=2 l_{i} / l_{i} \leq 2 l_{i}^{1} / L A^{R-1}$. If $L A^{R-1}+1 \leq l_{i} \leq 2 L A^{R-1}$, then $l_{i}^{L} \leq 3$, because $X^{\kappa}$ may start at an interface between two blocks $\widetilde{B}_{R}^{A}(x, k)$ and immediately jump from one such block to another. However, to afterwards reach the next block $\widetilde{B}_{R}^{A}(x, k)$ it has to cross at least $L A^{R-1} 1$-blocks, and so $l_{i}^{L} \leq 3 l_{i} / l_{i} \leq 3 l_{i}^{1} / L A^{R-1}$. Furthermore, for $j \in \mathbb{N}$, if $j L A^{R-1}+1 \leq l_{i} \leq(j+1) L A^{R-1}$, then

$$
l_{i}^{L} \leq(j+2) l_{i} / l_{i} \leq(j+2) l_{i} / j L A^{R-1} .
$$

Therefore we have

$$
k_{*}=\sum_{i=1}^{t / L A^{R-1}} l_{i} \geq \frac{L A^{R-1}}{3} \sum_{i=1}^{t / L A^{R-1}} l_{i}^{L},
$$

or $\sum_{i=1}^{t / L A^{R-1}} l_{i}^{L} \leq\left(3 / L A^{R-1}\right) k_{*}=l_{R}$, which completes the proof.

\section{Proof of Proposition 4.2}

In Section 6.1 we reduce the problem to one dimension and recall two discrete rearrangement inequalities from the literature (Propositions 6.3 6.4 below). In Section 6.2 we use the latter to give the proof of Proposition 4.2.

\subsection{Reduction to one dimension and discrete rearrangement inequalities}

Lemma 6.1. Let $B \subseteq \mathbb{Z}^{d} \times[0, t]$. Then, for all $C \geq 0$,

$$
E_{0}\left(e^{C l_{t}(B)}\right) \leq E_{0}\left(e^{C l_{t}\left(\pi_{1}(B)\right)}\right) .
$$

Proof. A $d$-dimensional simple random walk with jump rate $2 d \kappa$ is a vector of $d$ independent one-dimensional simple random walks, each having jump rate $2 \kappa$. Hence, given any set $B \subseteq$ $\mathbb{Z}^{d} \times[0, t]$,

$$
\forall s \geq 0: \quad X^{\kappa}(s) \in B \quad \Longrightarrow \quad \pi_{1}\left(X^{\kappa}\right)(s) \in \pi_{1}(B) .
$$

This in turn implies that $l_{t}(B) \leq l_{t}\left(\pi_{1}(B)\right)$, which proves the claim.

To prove Proposition 4.2 we need two discrete rearrangement inequalities [13], [14]. For an overview on continuous rearrangement inequalities we refer the reader to [12, Chapter 3].

Definition 6.2. A function $L: \mathbb{Z} \times \mathbb{Z} \rightarrow[0, \infty)$ is called of Riesz-type when, for all pairs of functions $f, g: \mathbb{Z} \rightarrow[0, \infty)$,

$$
\sum_{x, y \in \mathbb{Z}} f(x) L(x, y) g(y) \leq \sum_{x, y \in \mathbb{Z}} f^{\sharp}(x) L(x, y) g^{\sharp}(y) .
$$

Proposition 6.3. [13, Theorem 2.2], 14]) Let $K:[0, \infty) \rightarrow[0, \infty)$ be non-increasing. Then $L: \mathbb{Z} \times \mathbb{Z} \rightarrow[0, \infty)$ given by $L(x, y)=K(|x-y|)$ is of Riesz-type.

Note that $(x, y) \mapsto p_{s}^{\kappa}(x, y)$ with $p_{s}^{\kappa}(x, y)$ the transition kernel of one-dimensional simple random walk with jump rate $2 \kappa$ is of Riesz-type. Indeed, $p_{s}^{\kappa}(x, y)=p_{s}^{\kappa}(x-y, 0)=p_{s}^{\kappa}(|x-y|, 0)$ is a non-increasing function of $|x-y|$.

The following multiple-sum version of Proposition 6.3 will be needed also. 
Proposition 6.4. ([13, Lemma 9.1 in Chapter 2], [14]) Fix $n \in \mathbb{N}$. Let $L_{0}, L_{1}, \ldots, L_{n-1}$ be a collection of Riesz-type functions on $\mathbb{Z} \times \mathbb{Z}$, and let $S_{0}, S_{1}, \ldots, S_{n}$ be a collection of non-negative functions on $\mathbb{Z}$. Then

$$
\sum_{x_{0}, x_{1}, \ldots, x_{n} \in \mathbb{Z}}\left(\prod_{i=0}^{n-1} S_{i}\left(x_{i}\right) L_{i}\left(x_{i}, x_{i+1}\right)\right) S_{n}\left(x_{n}\right) \leq \sum_{x_{0}, x_{1}, \ldots, x_{n} \in \mathbb{Z}}\left(\prod_{i=0}^{n-1} S_{i}^{\sharp}\left(x_{i}\right) L_{i}\left(x_{i}, x_{i+1}\right)\right) S_{n}^{\sharp}\left(x_{n}\right) .
$$

\subsection{Proof of Proposition 4.2}

Proof. Let $\left(B_{R}\right)_{R \in \mathbb{N}}$ be a sequence in $\mathbb{Z} \times[0, t]$ (recall Lemma 6.1) and $\left(C_{R}\right)_{R \in \mathbb{N}}$ a sequence of nonnegative numbers. Write

$$
E_{0}\left(\exp \left\{\sum_{R \in \mathbb{N}} C_{R} l_{t}\left(B_{R}\right)\right\}\right)=\sum_{n \in \mathbb{N}_{0}} \frac{1}{n !} E_{0}\left(\left\{\sum_{R \in \mathbb{N}} C_{R} l_{t}\left(B_{R}\right)\right\}^{n}\right)
$$

The $n$-th moments in (6.5) may be rewritten as

$$
\sum_{R_{1}, \ldots, R_{n} \in \mathbb{N}}\left(\prod_{i=1}^{n} C_{R_{i}}\right) E_{0}\left(\prod_{i=1}^{n} l_{t}\left(B_{R_{i}}\right)\right)
$$

Write out

$$
\prod_{i=1}^{n} l_{t}\left(B_{R_{i}}\right)=\int_{0}^{t} d s_{1} \ldots \int_{0}^{t} d s_{n} \mathbb{1}\left\{X^{\kappa}\left(s_{1}\right) \in B_{R_{1}}, \ldots X^{\kappa}\left(s_{n}\right) \in B_{R_{n}}\right\},
$$

so that the second factor under the sum in (6.6) equals

$$
\int_{0}^{t} d s_{1} \ldots \int_{0}^{t} d s_{n} P_{0}\left(X^{\kappa}\left(s_{1}\right) \in B_{R_{1}}, \ldots X^{\kappa}\left(s_{n}\right) \in B_{R_{n}}\right) .
$$

Fix a choice of $\left(s_{1}, \ldots, s_{n}\right) \in[0, t]^{n}$, and let $B_{R_{s_{i}}}$ be the spatial part of $B_{R_{i}} \cap\left(\mathbb{Z} \times\left\{s_{i}\right\}\right)$. Without loss of generality we may assume that $s_{1}<s_{2}<\ldots<s_{n}$, so that the probability in (6.8) becomes $\left(x_{0}=0, s_{0}=0\right)$

$$
\sum_{x_{1}, \ldots, x_{n} \in \mathbb{Z}}\left(\prod_{i=0}^{n} \mathbb{1}\left\{x_{i} \in B_{R_{s_{i}}}\right\}\right)\left(\prod_{i=0}^{n-1} p_{s_{i+1}-s_{i}}^{\kappa}\left(x_{i}, x_{i+1}\right)\right) .
$$

An application of Proposition 6.4 gives that (6.9) is bounded from above by

$$
\sum_{x_{1}, \ldots x_{n} \in \mathbb{Z}}\left(\prod_{i=0}^{n} \mathbb{1}\left\{x_{i} \in B_{R_{s_{i}}}^{\sharp}\right\}\right)\left(\prod_{i=0}^{n-1} p_{s_{i+1}-s_{i}}^{\kappa}\left(x_{i}, x_{i+1}\right)\right),
$$

so that, by (6.8),

$$
E_{0}\left(\prod_{i=1}^{n} l_{t}\left(B_{R_{i}}\right)\right) \leq E_{0}\left(\prod_{i=1}^{n} l_{t}\left(B_{R_{i}}^{\sharp}\right)\right) .
$$

Inserting this back into (6.5) and (6.6), we get the claim. 


\section{Proof of Proposition 2.3}

In Section 7.1 we introduce some notation and state two more propositions, Propositions 7.3 7.4 below, whose proof is given in Sections 7.3 7.4. In Section 7.2 we give the proof of Proposition 2.3 subject to these propositions.

\subsection{Two more propositions}

Henceforth we assume that $\alpha$ in (1.13) takes the form $\alpha=4 M \kappa$ with $M$ a constant that will be determined later on. Recall the definition of $\pi_{1}$ below (2.2) and of $\bar{\xi}$ in (2.4).

Definition 7.1. The subpedestal of $B_{1}^{A, 4 M \kappa}(x, k)$ is (see Fig. 國)

$$
\begin{aligned}
B_{1, \mathrm{sub}}^{A, 4 M \kappa}(x, k)= & \left\{y \in \pi_{1}\left(B_{1}^{A, 4 M \kappa}(x, k)\right):\right. \\
& \left.|y(j)-z(j)| \geq 2 M \kappa A, j \in\{1,2, \ldots, d\} \forall z \in \partial \pi_{1}\left(B_{1}^{A, 4 M \kappa}(x, k)\right)\right\} \times\{k A\} .
\end{aligned}
$$

Definition 7.2. Let $\varepsilon>0$, and $k, n \in \mathbb{N}_{0}$ such that $n \geq k$. A block $B_{1}^{A, 4 M \kappa}(x, k)$ is called $\varepsilon$-sufficient at level $n$ when, for every $y \in \pi_{1}\left(B_{1, \text { sub }}^{A, 4 \kappa}(x, k)\right)$,

$$
E_{y}\left(\exp \left\{\int_{0}^{A} \bar{\xi}\left(X^{\kappa}(s), A(n-k)-s\right) d s\right\} \mathbb{1}\left\{N\left(X^{\kappa}, A\right) \leq M \kappa A\right\}\right) \leq e^{\varepsilon A} .
$$

Otherwise it is called $\varepsilon$-insufficient at level n. A subpedestal is called $\varepsilon$-(in)sufficient at level $n$ when its corresponding block is $\varepsilon$-(in)sufficient at level $n$.

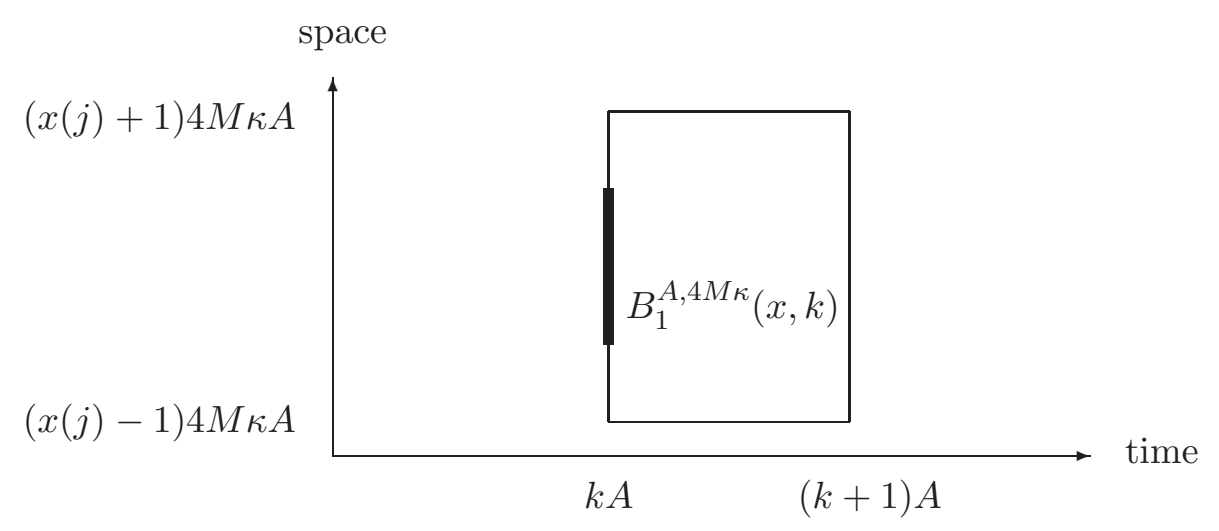

Figure 5: The thick line is the subpedestal.

Proposition 7.3. Let $A>1$. There is a constant $C_{3}>0$ such that for all $n \in \mathbb{N}$ the number of different sequences of subpedestals $B_{1, \text { sub }}^{A, 4 M \kappa}(0,0), B_{1, \text { sub }}^{A, 4 M \kappa}\left(x_{1}, 1\right), \ldots, B_{1, \text { sub }}^{A, 4 M \kappa}\left(x_{n-1}, n-1\right)$ with the property that there is a path $\Phi:[0, A n] \rightarrow \mathbb{Z}^{d}$ with at most $M \kappa A n$ jumps satisfying $\Phi(k A) \in$ $B_{1, \text { sub }}^{A, 4 M \kappa}\left(x_{k}, k\right), k \in\{0,1, \ldots, n-1\}$, is bounded from above by $e^{C_{3} n}$.

Proposition 7.4. Fix $\varepsilon>0$. Let $\delta=\frac{1}{4} \varepsilon$ in the definition of $\bar{\xi}$ and $A>1$. Then there is a $\kappa_{0}>0$ such that, for all $\kappa \geq \kappa_{0}$ and $\xi$-a.s. for all $n \in \mathbb{N}$, all blocks $B_{1}^{A, 4 M \kappa}(x, k), x \in \mathbb{Z}^{d}$, $k \in \mathbb{N}, k \leq n$, are $\varepsilon$-sufficient at level $n$. 


\subsection{Proof of Proposition 2.3 subject to two propositions}

Proof. The proof comes in 2 Steps.

1. Fix $\varepsilon>0$ and put $\delta=\frac{1}{4} \varepsilon$. Choose $\kappa \geq \kappa_{0}$ according to Proposition 7.4. Then the tail estimate $P(\operatorname{POISSON}(\lambda) \geq k) \leq e^{-\lambda}(\lambda e)^{k} / k^{k}, k \geq 2 \lambda+1$, for Poisson-distributed random variables with mean $\lambda$ shows that, for $M>0$ large enough,

$$
\begin{aligned}
& E_{0}\left(\exp \left\{\int_{0}^{A n} \bar{\xi}\left(X^{\kappa}(s), A n-s\right) d s\right\} \mathbb{1}\left\{N\left(X^{\kappa}, A n\right)>M \kappa A n\right\}\right) \\
& \leq e^{2 \delta A^{d+1} n} e^{-2 d \kappa A n} \exp \{-M \kappa A n[\log (M / 2 d)-1]\},
\end{aligned}
$$

where we use (2.4). Since we later let $\kappa \rightarrow \infty,(7.3)$ shows that it is enough to concentrate on contributions coming from paths with at most $M \kappa A n$ jumps. To that end, fix a $\mathbb{Z}^{d}$-valued sequence of vertices $x_{0}, x_{1}, \ldots, x_{n-1}$ such that $x_{0}=0$ and such that there is a path that starts in 0 , makes $0 \leq j \leq M \kappa A n$ jumps, and is in the subpedestal $B_{1, \text { sub }}^{A, 4 M \kappa}\left(x_{k}, k\right)$ at time $k A$ for $k \in\{0,1, \ldots, n-1\}$. By the Markov property of $X^{\kappa}$ applied at times $k A, k \in\{1,2, \ldots, n-1\}$,

$$
\begin{aligned}
E_{0}\left(\exp \left\{\int_{0}^{A n} \bar{\xi}\left(X^{\kappa}(s), A n-s\right) d s\right\} \mathbb{1}\left\{N\left(X^{\kappa}, A n\right) \leq M \kappa A n\right\}\right. \\
\left.\quad \times \prod_{k=1}^{n-1} \mathbb{1}\left\{X^{\kappa}(k A) \in B_{1, \mathrm{sub}}^{A, 4 M \kappa}\left(x_{k}, k\right)\right\}\right) \\
\leq \prod_{k=0}^{n-1} \sup _{y \in \pi_{1}}\left(B_{1, \mathrm{sub}}^{A, 4 M \kappa}\left(x_{k}, k\right)\right) \\
E_{y}\left(\exp \left\{\int_{0}^{A} \bar{\xi}\left(X^{\kappa}(s), A(n-k)-s\right) d s\right\}\right) .
\end{aligned}
$$

This is at most

$$
\begin{aligned}
\prod_{k=0}^{n-1}\left[\sup _{y \in \pi_{1}\left(B_{1, \text { sub }}^{A, 4 M \kappa}\left(x_{k}, k\right)\right)} E_{y}\left(\exp \left\{\int_{0}^{A} \bar{\xi}\left(X^{\kappa}(s), A(n-k)-s\right) d s\right\} \mathbb{1}\left\{N\left(X^{\kappa}, A\right) \leq M \kappa A\right\}\right)\right. \\
\left.\quad+\sup _{y \in \pi_{1}\left(B_{1, \text { sub }}^{A, 4 M \kappa}\left(x_{k}, k\right)\right)} E_{y}\left(\exp \left\{\int_{0}^{A} \bar{\xi}\left(X^{\kappa}(s), A(n-k)-s\right) d s\right\} \mathbb{1}\left\{N\left(X^{\kappa}, A\right)>M \kappa A\right\}\right)\right] \\
=\sum_{J \subset\{0,1, \ldots, n-1\}}[ \\
\prod_{k \in J} \sup _{y \in \pi_{1}\left(B_{1, \text { sub }}^{A, 4 M \kappa}\left(x_{k}, k\right)\right)} E_{y}\left(\exp \left\{\int_{0}^{A} \bar{\xi}\left(X^{\kappa}(s), A(n-k)-s\right) d s\right\} \mathbb{1}\left\{N\left(X^{\kappa}, A\right) \leq M \kappa A\right\}\right) \\
\left.\times \prod_{k \notin J} \sup _{y \in \pi_{1}\left(B_{1, \text { sub }}^{A, 4 M \kappa}\left(x_{k}, k\right)\right)} E_{y}\left(\exp \left\{\int_{0}^{A} \bar{\xi}\left(X^{\kappa}(s), A(n-k)-s\right) d s\right\} \mathbb{1}\left\{N\left(X^{\kappa}, A\right)>M \kappa A\right\}\right)\right] .
\end{aligned}
$$

Now, by the Poisson tail estimate mentioned above and the fact that $\bar{\xi}<2 \delta A^{d}$, the second factor under the sum in (7.5) may be bounded from above by

$$
\left(e^{2 \delta A^{d+1}} e^{-2 d \kappa A} \exp \{-M \kappa A[\log (M / 2 d)-1]\}\right)^{n-|J|} \text {. }
$$


Since, by Proposition 7.4 and our choice of $\kappa$ (see the observation made prior to (7.3)), all blocks $B_{1}^{A, 4 M \kappa}(x, k), x \in \mathbb{Z}^{d}, k \in \mathbb{N}_{0}, k \leq n$, are $\varepsilon$-sufficient at level $n$, we may conclude that all $y \in \pi_{1}\left(B_{1, \text { sub }}^{A, 4 M \kappa}\left(x_{k}, k\right)\right)$ with $k \in J$ are in an $\varepsilon$-sufficient subpedestal at level $n$. Hence, using the binomial formula, we may estimate (7.5) from above by

$$
\begin{aligned}
\sum_{J \subset\{0,1, \ldots, n-1\}} e^{A \varepsilon|J|}\left(e^{2 \delta A^{d+1}} e^{-2 d \kappa A} \exp \{-M \kappa A[\log (M / 2 d)-1]\}\right)^{n-|J|} \\
=\left(e^{A \varepsilon}+e^{2 \delta A^{d+1}} e^{-2 d \kappa A} \exp \{-M \kappa A[\log (M / 2 d)-1]\}\right)^{n} .
\end{aligned}
$$

2. Summing over all possible sequences $\left(x_{i}\right)_{i \in\{1,2, \ldots, n-1\}}$ compatible with a path $\Phi$ such that $\Phi(0)=0$ and $N(\Phi, A n) \leq M \kappa A n$, and using (7.4 [7.7) and Proposition 7.3, we obtain

$$
\begin{aligned}
& E_{0}\left(\exp \left\{\int_{0}^{A n} \bar{\xi}\left(X^{\kappa}(s), A n-s\right) d s\right\} \mathbb{1}\left\{N\left(X^{\kappa}, A n\right) \leq M \kappa A n\right\}\right) \\
& \leq \sum_{x_{1}, x_{2}, \ldots, x_{n-1}} E_{0}\left(\exp \left\{\int_{0}^{A n} \bar{\xi}\left(X^{\kappa}(s), A n-s\right) d s\right\} \mathbb{1}\left\{N\left(X^{\kappa}, A n\right) \leq M \kappa A n\right\}\right. \\
& \left.\times \prod_{k=1}^{n-1} \mathbb{1}\left\{X^{\kappa}(k A) \in B_{1, \text { sub }}^{A, 4 M \kappa}\left(x_{k}, k\right)\right\}\right) \\
& \leq \sum_{x_{1}, x_{2}, \ldots, x_{n-1}} \prod_{k=0}^{n-1} \sup _{y \in B_{1, \text { sub }}^{A, 4 M \kappa}\left(x_{k}, k\right)} E_{y}\left(\exp \left\{\int_{0}^{A n} \bar{\xi}\left(X^{\kappa}(s), A n-s\right) d s\right\}\right) \\
& \leq e^{C_{3} n}\left(e^{A \varepsilon}+e^{2 \delta A^{d+1}} e^{-2 d \kappa A} \exp \{-M \kappa A[\log (M / 2 d)-1]\}\right)^{n} .
\end{aligned}
$$

Combining (7.3 (7.8), we get

$$
\begin{aligned}
& \limsup _{\kappa \rightarrow \infty} \limsup _{n \rightarrow \infty} \frac{1}{A n} \log E_{0}\left(\exp \left\{\int_{0}^{t} \bar{\xi}\left(X^{\kappa}(s), t-s\right) d s\right\}\right) \\
& \leq \frac{C_{3}}{A}+\frac{1}{A} \limsup _{\kappa \rightarrow \infty} \log \left(e^{A \varepsilon}+e^{2 \delta A^{d+1}} e^{-2 d \kappa A} \exp \{-M \kappa A[\log (M / 2 d)-1]\}\right)=\frac{C_{3}}{A}+\varepsilon
\end{aligned}
$$

Since $\varepsilon=4 \delta$, this yields the claim.

\subsection{Proof of Proposition $\mathbf{7 . 3}$}

Proof. Write $\|\cdot\|$ for the $\ell^{1}$-norm on $\mathbb{Z}^{d}$. Let $B_{1, \text { sub }}^{A, 4 M \kappa}(0,0), B_{1, \text { sub }}^{A, 4 M \kappa}\left(x_{1}, 1\right), \ldots, B_{1, \text { sub }}^{A, 4 M \kappa}\left(x_{n-1}\right.$, $n-1)$ be a sequence of subpedestals that may be crossed by a path $\Phi$ with at most $M \kappa A n$ jumps. Since $\Phi$ needs at least $\left(\left\|x_{k}-x_{k-1}\right\|-d\right)_{+} 4 M \kappa A$ jumps to go from $B_{1, \text { sub }}^{A, 4 M \kappa}\left(x_{k-1}, k-1\right)$ to $B_{1 \text {,sub }}^{A, 4 M \kappa}\left(x_{k}, k\right), k \in\{1,2, \ldots, n-1\}$, we obtain the bound

$$
\sum_{k=1}^{n-1}\left(\left\|x_{k}-x_{k-1}\right\|-d\right)_{+} \leq \frac{M \kappa A n}{4 M \kappa A}=\frac{n}{4},
$$

which implies that

$$
\sum_{k=1}^{n-1}\left\|x_{k}-x_{k-1}\right\| \leq \frac{(1+4 d) n}{4} .
$$


As shown in Hardy and Ramanujan [7] and Erdös [2], there are $a, b>0$ such that the number of integer-valued sequences $\left(a_{k}\right)_{k \in \mathbb{N}}$ such that $\sum_{k \in \mathbb{N}} a_{k} \leq(1+4 d) n / 4$ is bounded from above by $a n e^{b \sqrt{n}}$. To conclude, define $a_{k}=\left\|x_{k}-x_{k-1}\right\|, k \in\{1,2, \ldots, n-1\}$, and note that the sequence $\left(a_{k}\right)_{k \in\{1,2, \ldots, n-1\}}$ determines the sequence $\left(x_{k}\right)_{k \in\{0,1, \ldots, n-1\}}$ uniquely when it is known for all $k \in\{1,2, \ldots, n-1\}$ and all $j \in\{1,2, \ldots, d\}$ whether $x_{k}(j)-$ $x_{k-1}(j)$ is positive, zero or negative. Consequently, the number of different subpedestals $B_{1, \text { sub }}^{A, 4 M \kappa}(0,0), B_{1, \text { sub }}^{A, 4 M}\left(x_{1}, 1\right), \ldots, B_{1, \text { sub }}^{A, 4 M \kappa}\left(x_{n-1}, n-1\right)$ that may be crossed by a path $\Phi$ with at most $M \kappa A n$ jumps is bounded from above by $3^{d n} a n e^{b \sqrt{n}} \leq e^{C_{3} n}$ for some $C_{3}>0$.

\subsection{Proof of Proposition 7.4}

The proof of Proposition 7.4 is given in Section 7.5 subject to Lemmas 7.5 7.6 below, which are stated in Sections 7.4.17 7.4.2. The proof of the first lemma is given in Section 7.4.1, the proof of the second lemma is deferred to Appendix B

\subsubsection{A time-dependent Feynman-Kac estimate}

Recall (2.4). Abbreviate

$$
Q^{\kappa \log \kappa}=(-\kappa \log \kappa, \kappa \log \kappa)^{d} \cap \mathbb{Z}^{d} .
$$

Lemma 7.5. Fix $A>1$ and $m>0$ such that $A m \in \mathbb{N}$. There is a $\kappa_{0}=\kappa_{0}(M, A)$ such that, $\xi$-a.s. for all $x \in \mathbb{Z}^{d}$,

$$
\log E_{x}\left(\exp \left\{\int_{0}^{A} \bar{\xi}\left(X^{\kappa}(s), A-s\right) d s\right\} \mathbb{1}\left\{N\left(X^{\kappa}, A\right) \leq M \kappa A\right\}\right) \leq \frac{\kappa}{m} \sum_{k=1}^{A m} \lambda_{1}\left(\bar{\xi}_{k} / \kappa\right), \quad \kappa \geq \kappa_{0},
$$

where $\lambda_{1}\left(\bar{\xi}_{k} / \kappa\right)$ is the top of the spectrum of the operator $\Delta+\frac{1}{\kappa} \sup _{r \in[(k-1) / m, k / m)} \bar{\xi}(\cdot, r)$, $k \in\{1,2, \ldots, A m\}$.

Proof. We give the proof for $x=0$. The proof for $x \in \mathbb{Z}^{d} \backslash\{0\}$ goes along the same lines. First note that we may rewrite the expectation in the left-hand side of (17.13) as

$$
E_{0}\left(\exp \left\{\frac{1}{\kappa} \int_{0}^{\kappa A} \bar{\xi}(X(s), A-s / \kappa) d s\right\} \mathbb{1}\{N(X, \kappa A) \leq M \kappa A\}\right),
$$

where $X$ is simple random walk with step rate $2 d$. Furthermore, there is a $\kappa_{0}=\kappa_{0}(M, A)$ such that $M \kappa A \leq \kappa \log \kappa$ for all $\kappa \geq \kappa_{0}$. Hence, by the Markov property of $X$ applied at times $k \kappa / m, k \in\{1,2, \ldots, A m\}$, we may estimate (7.14) from above by

$$
\begin{aligned}
& E_{0}\left(\exp \left\{\frac{1}{\kappa} \int_{0}^{\kappa A} \bar{\xi}(X(s), A-s / \kappa) d s\right\} \mathbb{1}\left\{X([0, \kappa A]) \subseteq Q^{\kappa \log \kappa}\right\}\right) \\
& \leq \prod_{k=1}^{A m} \sup _{\substack{x \in \mathbb{Z}^{d} \\
\|x\|_{\infty}<\kappa \log \kappa}} E_{x}\left(\exp \left\{\frac{1}{\kappa} \int_{0}^{\kappa / m} \bar{\xi}(X(s), k / m-s / \kappa) d s\right\} \mathbb{1}\left\{X([0, \kappa / m]) \subseteq Q^{\kappa \log \kappa}\right\}\right) .
\end{aligned}
$$

Next, for $k \in\{1,2, \ldots, A m\}$ define

$$
\bar{\xi}_{k}(x)=\sup _{r \in[(k-1) / m, k / m)} \bar{\xi}(x, r), \quad x \in \mathbb{Z}^{d} .
$$


Then (7.15) is at most

$$
\prod_{k=1}^{A m} \sup _{x \in \mathbb{Z}^{d} / /\|x\|_{\infty}<\kappa \log \kappa} E_{x}\left(\exp \left\{\frac{1}{\kappa} \int_{0}^{\kappa / m} \bar{\xi}_{k}(X(s)) d s\right\} \mathbb{1}\left\{X([0, \kappa / m]) \subseteq Q^{\kappa \log \kappa}\right\}\right) .
$$

For each $k \in\{1,2, \ldots, A m\}$, each expectation under the product in (17.17) is a solution of the equation

$$
\left\{\begin{array}{l}
\frac{\partial u_{k}}{\partial t}(x, t)=\left[\left(\Delta+\frac{1}{\kappa} \bar{\xi}_{k}(x)\right) u_{k}\right](x, t), \quad\|x\|_{\infty}<\kappa \log \kappa, t \geq 0, \\
u_{k}(x, 0)=1,
\end{array}\right.
$$

with Dirichlet boundary conditions evaluated at time $\kappa / m$. However, on any finite subset of $\mathbb{Z}^{d}$ the operator $\Delta+\frac{1}{\kappa} \bar{\xi}_{k}$ is a self-adjoint matrix. Therefore, by the spectral representation theorem, we may rewrite each expectation under the product in (7.17) as

$$
\sum_{j=1}^{\left|Q^{\kappa \log \kappa}\right|} e^{(\kappa / m) \lambda_{j}^{D}\left(\bar{\xi}_{k} / \kappa\right)}\left\langle v_{j}^{k}, \mathbb{1}_{Q^{\kappa \log \kappa} \kappa}\right\rangle v_{j}^{k}(x)
$$

where $\lambda_{j}^{D}\left(\bar{\xi}_{k} / \kappa\right)$ is the $j$-th largest eigenvalue of $\Delta+\bar{\xi}_{k} / \kappa$ with Dirichlet boundary conditions on $Q^{\kappa \log \kappa}, j \in\left\{1,2, \ldots,\left|Q^{\kappa \log \kappa}\right|\right\}$, and the $v_{j}^{k}, j \in\left\{1,2, \ldots,\left|Q^{\kappa \log \kappa}\right|\right\}$, form an orthonormal system of eigenvectors such that, for all $k \in\{1,2, \ldots, A m\}$,

$$
\mathbb{R}^{\left|Q^{\kappa \log \kappa}\right|}=\operatorname{ker}\left(e^{\Delta+\bar{\xi}_{k} / \kappa}\right) \oplus \operatorname{span}\left\{v_{j}^{k}, j \in\left\{1,2, \ldots,\left|Q^{\kappa \log \kappa}\right|\right\}\right\}
$$

(Since $e^{\Delta+\bar{\xi}_{k} / \kappa}$ is a strictly positive operator, $\operatorname{ker}\left(e^{\Delta+\bar{\xi}_{k} / \kappa}\right)=\{0\}$.) In particular, for each $k \in\{1,2, \ldots, A m\}$ there is a sequence of real-valued numbers $\left(\mu_{j}^{k}\right)_{j \in\left\{1,2, \ldots,\left|Q^{\kappa \log \kappa}\right|\right\}}$ such that $\sum_{j=1}^{k} \mu_{j}^{k} v_{j}^{k}=\mathbb{1}_{Q^{\kappa} \log \kappa}$. Inserting the above representation of $\mathbb{1}_{Q^{\kappa \log \kappa}}$ into (17.19), we see that (7.19) is bounded from above by

$$
e^{(\kappa / m) \lambda_{1}^{D}\left(\bar{\xi}_{k} / \kappa\right)} .
$$

Combining (7.14)-(7.21), we get

$$
\log E_{x}\left(\exp \left\{\int_{0}^{A} \bar{\xi}\left(X^{\kappa}(s), A-s\right) d s\right\} \mathbb{1}\left\{N\left(X^{\kappa} ; A\right) \leq M \kappa A\right\}\right) \leq \frac{\kappa}{m} \sum_{k=1}^{A m} \lambda_{1}^{D}\left(\bar{\xi}_{k} / \kappa\right) .
$$

Finally, by the Rayleigh-Ritz principle we have that $\lambda_{1}^{D}\left(\bar{\xi}_{k} / \kappa\right) \leq \lambda_{1}\left(\bar{\xi}_{k} / \kappa\right)$, where $\lambda_{1}\left(\bar{\xi}_{k} / \kappa\right)$ is the top of the spectrum of $\Delta+\bar{\xi}_{k} / \kappa$.

\subsubsection{A spectral estimate}

Let $(B(x))_{x \in \mathbb{Z}^{d}}$ be an arbitrary partition of $\mathbb{Z}^{d}$ into finite boxes. Let $\langle\cdot, \cdot\rangle$ be the scalar product on $\mathbb{R}^{B}$ and on $\ell^{2}\left(\mathbb{Z}^{d}\right)$. Let $V: \mathbb{Z}^{d} \rightarrow \mathbb{R}$ be bounded such that there is a $\delta>0$ for which

$$
\frac{1}{|B(x)|} \sum_{y \in B(x)} V(y) \leq 2 \delta \quad x \in \mathbb{Z}^{d} .
$$

The proof of the following lemma is deferred to Appendix B. 
Lemma 7.6. Subject to (7.23), there is a $\kappa_{0}>0$ such that, for all $\kappa \geq \kappa_{0}$,

$$
\sup _{\substack{f \in l^{2}\left(\mathbb{Z}^{d}\right) \\\|f\|_{2}=1}}\left\langle\left(\Delta+\frac{1}{\kappa} V\right) f, f\right\rangle \leq 4 \frac{1}{\kappa} \delta .
$$

Lemma 7.6 and the Rayleigh-Ritz principle yield that the top of the spectrum of $\Delta+\frac{1}{\kappa} V$ is bounded from above by $4 \frac{1}{\kappa} \delta$ for $\kappa \geq \kappa_{0}$.

\subsection{Completion of the proof of Proposition 7.4}

Proof. Fix $\delta>0, A>1$ and $m>1$. By Lemma 7.5, there is a $\kappa_{0}>0$ such that, for all $\kappa \geq \kappa_{0}$ and $\xi$-a.s. for all $x \in \mathbb{Z}^{d}$,

$$
\begin{gathered}
\log E_{x}\left(\exp \left\{\int_{0}^{A} \bar{\xi}\left(X^{\kappa}(s), A-s\right) d s\right\} \mathbb{1}\left\{N\left(X^{\kappa} ; A\right) \leq M \kappa A\right\}\right) \\
\leq \frac{\kappa}{m} \sum_{k=1}^{A m} \lambda_{1}\left(\bar{\xi}_{k} / \kappa\right), \quad \kappa \geq \kappa_{0}=\kappa_{0}(M, A) .
\end{gathered}
$$

Next, by Lemma 7.6 with $V=\bar{\xi}_{k}, k \in\{1,2, \ldots, A m\}$ (recall (17.16) $)$ and $B(x)=\pi_{1}\left(B_{1}^{A}(x, 0)\right.$ ) (recall (1.13); $\pi_{1}$ denotes the projection onto the spatial coordinates), there is a $\kappa_{1}>0$ such that, for all $\kappa \geq \kappa_{1}$ and all $k \in\{1,2, \ldots, A m\}$,

$$
\lambda_{1}\left(\bar{\xi}_{k} / \kappa\right) \leq 4 \frac{1}{\kappa} \delta .
$$

This shows that, $\xi$-a.s. for $\kappa \geq \max \left\{\kappa_{0}, \kappa_{1}\right\}$, any block $B_{1}^{A, 4 M \kappa}(x, 0), x \in \mathbb{Z}^{d}$, is $\varepsilon$-sufficient at level 1 . The stationarity of $\xi$ in time completes the proof.

\section{A Proof of Lemmas 4.34 .4}

In this section we prove two lemmas that were used in Section 4

\section{A.1 Proof of Lemma 4.3}

Proof. Our first observation is that

$$
\limsup _{t \rightarrow \infty} \frac{1}{t} \log E_{0}\left(\exp \left\{\sum_{i=1}^{n} \beta_{i} \sum_{x \in I_{i}} l_{t}\left(X^{\kappa}, x\right)\right\}\right) \leq \mu,
$$

where

$$
\mu=\sup _{\substack{f \in l^{2}(\mathbb{Z}): \\\|f\|_{2}=1, f \geq 0}} \mu(f)
$$

with

$$
\mu(f)=\left(f,\left[\kappa \Delta+\sum_{i=1}^{n} \beta_{i} \mathbb{1}_{I_{i}}\right] f\right)=\sum_{i=1}^{n} \beta_{i} \sum_{x \in I_{i}} f^{2}(x)-\kappa \sum_{x \in \mathbb{Z}}[f(x+1)-f(x)]^{2} .
$$


Indeed, this follows from the large deviation principle for the occupation time measure of one-dimensional simple random walk on $\mathbb{Z}$ with jump rate $2 \kappa$ (which is the continuous-time Markov process with generator $\kappa \Delta$ ) in combination with Varadhan's lemma (see [9, Chapters $3-4]$ ). A formal proof proceeds by truncating $\mathbb{Z}$ to a large finite torus, wrapping the random walk around the torus, deriving the claim for a fixed torus size, letting the torus size tend to infinity, and showing that the variational formula on the finite torus converges to the variational formula on $\mathbb{Z}$. The details are standard and are left to the reader (see [9, Chapter $8])$.

We claim that $\mu$ is the largest eigenvalue of $\kappa \Delta+\sum_{i=1}^{n} \beta_{i} \mathbb{1}_{I_{i}}$. Indeed, by [8, Theorem 2.2] the operator $\kappa \Delta+\sum_{i=1}^{n} \beta_{i} \mathbb{1}_{I_{i}}$ has at least one eigenvalue. Consequently, the min-max principle [15, Theorem XIII.1] yields the claim. The inequality in (4.21) now follows from [17, Corollary 1.4].

\section{A.2 Proof of Lemma 4.4}

Proof. Let $t, \kappa>0$ and let $X^{\kappa}$ be one-dimensional random walk with step rate $2 \kappa>0$. We first show that for all $C>0$,

$$
P_{0}\left(\sup _{0 \leq s \leq t}\left|X^{\kappa}(s)\right| \geq C \sqrt{\kappa t}\right) \leq 2 e^{-\frac{C^{2} \sqrt{\kappa t}}{2(C+3 \sqrt{\kappa t})}}
$$

The proof is based on a discretization argument in combination with Bernstein's inequality. Fix $n \in \mathbb{N}$ with $n \gg \kappa$, and define

$$
q^{n}(y)= \begin{cases}1-\frac{2 \kappa}{n}, & y=0 \\ \frac{\kappa}{n}, & y= \pm 1 \\ 0, & \text { elsewhere. }\end{cases}
$$

Let $X^{(n)}=\left(X^{(n)}(t)\right)_{t>0}$ be the discrete-time random walk with jump distribution $q^{n}$ and jump times $k / n, k \in \mathbb{N}$. Then, for each $t>0,\left(X^{(n)}(s)\right)_{0 \leq s \leq t}$ converges weakly as $n \rightarrow \infty$ to $\left(X^{\kappa}(s)\right)_{0 \leq s \leq t}$ in the Skorokhod space $D([0, t], \mathbb{Z})$. Since $X^{(n)}$ is unlikely to move in a short time interval, uniformly in $n$, it is enough to prove (A.4) for $X^{(n)}$ with $n$ fixed. To that end, let $k \in \mathbb{N}$ be such that $k / n \leq t<(k+1) / n$. Note that, because $X^{(n)}$ is a martingale, Doob's maximal inequality and Bernstein's inequality yield

$$
P_{0}\left(\sup _{0 \leq s \leq t} X^{(n)}(s) \geq C \sqrt{\kappa t}\right) \leq \exp \left\{-\frac{C^{2} \kappa t}{2(C \sqrt{\kappa t}+3 \kappa t)}\right\} \quad \forall C>0 .
$$

The same inequality is valid for the probability of $\sup _{0 \leq s \leq t}\left[-X^{(n)}(s)\right] \geq C \sqrt{\kappa t}$, which yields the claim in (A.4).

Next, note that if $X^{\kappa}$ leaves the spatial part of a space-time block $B_{1}(x, k ; \kappa)$, then there is at least one coordinate $j \in\{1, \ldots, d\}$ such that $\pi_{j}\left(X^{\kappa}(s)\right) \notin[\sqrt{\kappa} x(j) A, \sqrt{\kappa}(x(j)+1) A)$ for some $s \in[k A,(k+1) A)$, where $\pi_{j}\left(X^{\kappa}\right)$ denotes the projection of $X^{\kappa}$ onto the $j$-th coordinate. In particular, if $X^{\kappa}$ visits $l_{i}^{\kappa}(\kappa, 1)$-blocks with $l_{i}^{\kappa}>d$ in the time interval $[(i-1) A, i A)$, then there is at least one coordinate that visits at least $l_{i}^{\kappa} / d$ one-dimensional $(\kappa, 1)$-blocks, i.e., blocks of the form $[\sqrt{\kappa} x A, \sqrt{\kappa}(x+1) A) \times[k A,(k+1) A), x \in \mathbb{Z}$. Consequently, without loss of generality we may assume that $X^{\kappa}$ is one-dimensional simple random walk with step rate $2 \kappa$. 
Given $l_{1}^{\kappa}, \ldots, l_{t / A}^{\kappa} \in \mathbb{N}$, we say that $X^{\kappa}$ has label $\left(l_{1}^{\kappa}, \ldots, l_{t / A}^{\kappa}\right)$ when $X^{\kappa}$ crosses $l_{i}^{\kappa}(\kappa, 1)$-blocks in the time interval $[(i-1) A, i A), 1 \leq i \leq t / A$.

Next, fix $C_{7}>0$ and let $k_{*}(\kappa) \geq C_{7} t$, and note that

$$
P_{0}\left(X^{\kappa} \text { crosses } k_{*}(\kappa)(\kappa, 1) \text {-blocks }\right)=\sum_{\substack{\left.l_{i}^{\kappa}\right)_{i=1}^{t / A} \\ \sum l_{i}^{\kappa}=k_{*}(\kappa)}} P_{0}\left(X^{\kappa} \text { has label }\left(l_{1}^{\kappa}, \ldots, l_{t / A}^{\kappa}\right)\right) .
$$

Using the Markov property and the fact that a path crossing $l_{i}^{\kappa}(\kappa, 1)$-blocks has to travel a distance at least $\left(l_{i}^{\kappa}-2\right) \sqrt{\kappa} A / 2$, we may further estimate each summand in the right-hand side of (A.7) by

$$
\begin{aligned}
& E_{0}\left(\mathbb{1}\left\{X^{\kappa} \text { has label }\left(l_{1}^{\kappa}, \ldots, l_{t / A-1}^{\kappa}\right)\right\} P_{X^{\kappa}(t-A)}\left(X^{\kappa} \text { has label } l_{t / A}^{\kappa}\right)\right) \\
& \leq E_{0}\left(\mathbb{1}\left\{X^{\kappa} \text { has label }\left(l_{1}^{\kappa}, \ldots, l_{t / A-1}^{\kappa}\right)\right\} P_{X^{\kappa}(t-A)}\left(\sup _{0 \leq s \leq A}\left|X^{\kappa}(s)\right| \geq\left(l_{i}^{\kappa}-2\right) \sqrt{\kappa} A / 2\right)\right) .
\end{aligned}
$$

To proceed, note that, by (A.4),

$$
P_{X^{\kappa}(t-A)}\left(\sup _{0 \leq s \leq A}\left|X^{\kappa}(s)\right| \geq\left(l_{i}^{\kappa}-2\right) \sqrt{\kappa} A / 2\right) \leq \begin{cases}2 \exp \left\{-\frac{1}{4} \frac{\left(l_{i}^{\kappa}-2\right)^{2} A \sqrt{\kappa A}}{\left(l_{i}^{\kappa}-2\right) \sqrt{A}+6 \sqrt{\kappa A}}\right\}, & l_{i}^{\kappa} \geq 3 \\ 1, & l_{i}^{\kappa} \leq 2 .\end{cases}
$$

An iteration of the estimates in (A.8) yields that the left-hand side of (A.7) is bounded from above by

$$
\sum_{\substack{\left(l_{i}^{\kappa}\right)_{i=1}^{t / A}: \\ \sum l_{i}^{k}=k_{*}(\kappa)}} \prod_{\substack{i=1 \\ l=3}}^{t / A} 2 \exp \left\{-\frac{1}{4} \frac{\left(l_{i}^{\kappa}-2\right)^{2} A \sqrt{\kappa A}}{\left(l_{i}^{\kappa}-2\right) \sqrt{A}+6 \sqrt{\kappa A}}\right\}
$$

We have

$$
\sum_{\substack{i=1: \\ l_{i}^{\kappa} \geq 3}}^{t / A} \frac{\left(l_{i}^{\kappa}-2\right)^{2}}{\left(l_{i}^{\kappa}-2\right) \sqrt{A}+6 \sqrt{\kappa A}} \geq \sum_{\substack{i=1: \\ l_{i}^{\kappa} \geq 3}}^{t / A} \frac{\left(l_{i}^{\kappa}-2\right)}{\sqrt{A}+6 \sqrt{\kappa A}}
$$

Moreover,

$$
\sum_{\substack{i=1: \\ l_{i} \geq 3}}^{t / A}\left(l_{i}^{\kappa}-2\right) \geq k_{*}(\kappa)-\frac{4 t}{A} .
$$

Inserting (A.11 A.12) into (A.10), noting that 7, 2,

$$
\exists a, b>0: \text { number of summands in right-hand side of }\left(\text { A.7) } \leq a e^{b \sqrt{k_{*}(\kappa)}} / k_{*}(\kappa),\right.
$$

and choosing $C_{7}$ and $\kappa$ large enough $\left(C_{7}>4\right.$ is sufficient), we get that the right-hand side of (A.7) is at most $e^{-C_{8} A k_{*}(\kappa)}$. Here, $C_{8}$ is such that for all $k_{*}(\kappa) \geq C_{7} / A$ the inequality $\left(k_{*}(\kappa)-4 t / A\right) /(1 / \sqrt{\kappa}+6) \geq C_{8} k_{*}(\kappa)$ holds, which for $\kappa \geq 1$ is fulfilled when $C_{7}\left(1-7 C_{8}\right) \geq 4$. This yields the claim in (4.22). 


\section{B Proof of Lemma 7.6}

In Sections B.1 B.3 we prove a lemma that was used in Section 7 The proof is inspired by [1, Theorem 12].

\section{B.1 Neumann boundary conditions}

In this section we recall the definition and some properties of the discrete Laplacian with Neumann boundary conditions. For further details we refer the reader to [11.

Fix $x \in \mathbb{Z}^{d}, A>1$ and define the matrix $M_{B(x)}$ as

$$
M_{B(x)}(y, z)=\left\{\begin{array}{l}
1, \text { if } y, z \in B(x),\|y-z\|=1 \\
0, \text { otherwise }
\end{array}\right.
$$

and the number of neighbors of $y$ in $B(x)$ as

$$
n_{B(x)}(y)=|\{z \in B(x):\|y-z\|=1\}| .
$$

Definition B.1. The Neumann Laplacian $\Delta_{B(x)}$ on $B(x)$ is defined via the formula

$$
\Delta_{B(x)}=M_{B(x)}-n_{B(x)},
$$

where $n_{B(x)}$ is the multiplication operator with the function $n_{B(x)}$.

Remark B.2. The quadratic form associated with $\Delta_{B(x)}$ is given by

$$
\left\langle\Delta_{B(x)} f, g\right\rangle=-\frac{1}{2} \sum_{\substack{y, z \in B(x) \\\|y-z\|=1}}[f(y)-f(z)][g(y)-g(z)], \quad f, g \in \mathbb{R}^{B(x)} .
$$

$\left(\Delta_{B(x)}\right.$ does not see that $B(x)$ is imbedded in $\mathbb{Z}^{d}$, which is why it is sometimes referred to as the graph Laplacian on $B(x)$.)

Lemma B.3. The following properties hold for all $x \in \mathbb{Z}^{d}$ and $A>1$.

(a) $\left\langle\Delta_{B(x)} f, f\right\rangle \leq 0$ for all $f \in \mathbb{R}^{B(x)}$.

(b) $\Delta_{B(x)}$ is self-adjoint.

(c) $\operatorname{ker}\left(\Delta_{B(x)}\right)=\mathbb{R} \mathbb{1}$, where $\mathbb{1}$ is the vector in $\mathbb{R}^{B(x)}$ with all entries equal to one.

(d) For all $f \in \ell^{2}\left(\mathbb{Z}^{d}\right)$,

$$
\langle\Delta f, f\rangle \leq \sum_{x \in \mathbb{Z}^{d}}\left\langle\Delta_{B(x)} f^{B(x)}, f^{B(x)}\right\rangle,
$$

where $f^{B(x)}$ is the restriction of $f$ to $B(x)$.

Proof. Fix $x \in \mathbb{Z}^{d}$ and $A>1$.

(a) and (b) are consequences of Remark B.2.

(c) From Remark B.2 it is clear that constant functions are in the kernel of $\Delta_{B(x)}$. For the reverse direction, let $f \in \operatorname{ker}\left(\Delta_{B(x)}\right)$. Again by Remark B.2,

$$
0=\left\langle\Delta_{B(x)} f, f\right\rangle=-\frac{1}{2} \sum_{\substack{y, z \in B(x) \\\|y-z\|=1}}[f(y)-f(z)]^{2} .
$$


Hence, for all $y \in B(x)$ we have that $f(y)=f(z)$ for all $z$ such that $\|y-z\|=1, z \in B(x)$. (d) Let $f \in \ell^{2}\left(\mathbb{Z}^{d}\right)$. Then

$$
\begin{aligned}
-2\langle\Delta f, f\rangle & =\sum_{\substack{y, z \in \mathbb{Z}^{d} \\
\|y-z\|=1}}[f(y)-f(z)]^{2}=\sum_{x \in \mathbb{Z}^{d}} \sum_{y \in B(x)} \sum_{\substack{z \in \mathbb{Z}^{d} \\
\|y-z\|=1}}[f(y)-f(z)]^{2} \\
& \geq \sum_{x \in \mathbb{Z}^{d}} \sum_{y \in B(x)} \sum_{\substack{z \in B(x) \\
\|y-z\|=1}}[f(y)-f(z)]^{2}=-2 \sum_{x \in \mathbb{Z}^{d}}\left\langle\Delta_{B(x)} f^{B(x)}, f^{B(x)}\right\rangle,
\end{aligned}
$$

where the second equality uses that $(B(x))_{x \in \mathbb{Z}^{d}}$ is a partition of $\mathbb{Z}^{d}$, while the third equality follows from Remark B.2

\section{B.2 Proof of Lemma 7.6 subject to a further lemma}

Let $\|\cdot\|_{2}$ stand for both the Euclidean norm on $\mathbb{R}^{B}$ and the $\ell^{2}$-norm on $\ell^{2}\left(\mathbb{Z}^{d}\right)$.

Lemma B.4. Subject to (7.23), there is a $\kappa_{0}>0$ such that, for all $\kappa \geq \kappa_{0}$, all $f \in \mathbb{R}^{B(x)}$ and all $x \in \mathbb{Z}^{d}$,

$$
\left\langle\left(\Delta_{B(x)}+\frac{1}{\kappa} V\right) f, f\right\rangle \leq 4 \frac{1}{\kappa} \delta\|f\|_{2}^{2} .
$$

The proof of Lemma B.4 is deferred to Section B.3. First we complete the proof of Lemma 7.6 subject to Lemma B.4.

Proof. Let $f \in \ell^{2}\left(\mathbb{Z}^{d}\right)$ and $\kappa \geq \kappa_{0}$, where $\kappa_{0}$ is chosen according to Lemma B.4. Then, by Lemma B.3(d) and the fact that $(B(x))_{x \in \mathbb{Z}^{d}}$ is a partition of $\mathbb{Z}^{d}$, we may estimate

$$
\left\langle\left(\Delta+\frac{1}{\kappa} V\right) f, f\right\rangle \leq \sum_{x \in \mathbb{Z}^{d}}\left\langle\left(\Delta_{B(x)}+\frac{1}{\kappa} V\right) f^{B(x)}, f^{B(x)}\right\rangle .
$$

Since $f=\sum_{x \in \mathbb{Z}^{d}} f^{B(x)}$, we have $\|f\|^{2}=\sum_{x \in \mathbb{Z}^{d}}\left\|f^{B(x)}\right\|_{2}^{2}$. Combining (B.8 $[$ B.9), we get the claim.

\section{B.3 Proof of Lemma B.4}

Proof. Fix $x \in \mathbb{Z}^{d}$ and $A>1$. First recall that, by Lemma $\mathbb{B . 3}(\mathrm{c}), \operatorname{ker}\left(\Delta_{B(x)}\right)=\mathbb{R} \mathbb{1}$, so that we can write each $f \in \mathbb{R}^{B(x)}$ as $f=\alpha \mathbb{1}+g, \alpha \in \mathbb{R}, g \in(\mathbb{R} \mathbb{1})^{\perp}$. Therefore, using that $\Delta_{B(x)}$ is self-adjoint, see Lemma B.3(b) and hence symmetric, we obtain that

$$
\left\langle\left(\Delta_{B(x)}+\frac{1}{\kappa} V\right) f, f\right\rangle=\left\langle\Delta_{B(x)} g, g\right\rangle+\frac{1}{\kappa}\left[\alpha^{2}\langle V \mathbb{1}, \mathbb{1}\rangle+2 \alpha\langle V \mathbb{1}, g\rangle+\langle V g, g\rangle\right] .
$$

Using that the unit sphere intersected with $(\mathbb{R} 1)^{\perp}$ is compact, that $\Delta_{B(x)}$ is negative on $(\mathbb{R} 1)^{\perp}$, see Lemma $\mathbb{B . 3}(\mathrm{a})$, and that the scalar product is continuous, we deduce that there is an $\eta>0$ such that $\left\langle\Delta_{B(x)} h, h\right\rangle \leq-\eta$ for all $h \in(\mathbb{R} \mathbb{1})^{\perp}$ with $\|h\|_{2}=1$. Hence we may estimate the right-hand side of (B.10) from above by

$$
-\eta\|g\|_{2}^{2}+\frac{1}{\kappa}\left[\alpha^{2}\langle V \mathbb{1}, \mathbb{1}\rangle+2 \alpha\langle V \mathbb{1}, g\rangle+\langle V g, g\rangle\right] .
$$


Next, by (7.23), we have $\langle V \mathbb{1}, \mathbb{1}\rangle=\sum_{y \in B(x)} V(y) \leq 2 \delta|B(x)|$. An additional application of the Cauchy-Schwarz inequality shows that (B.11) is at most

$$
-\eta\|g\|_{2}^{2}+\frac{1}{\kappa}\left[\alpha^{2} 2 \delta|B(x)|+2 \alpha\|V \mathbb{1}\|_{2}\|g\|_{2}+\|V g\|_{2}\|g\|_{2}\right] .
$$

Using the bound $\|V g\|_{2} \leq\|V\|_{\infty}\|g\|_{2}$ (which also holds for $g$ replaced by $\mathbb{1}$ ) and the assumption that $V$ is bounded, we may further estimate (B.12) from above by

$$
-\eta\|g\|_{2}^{2}+\frac{1}{\kappa}\left[\alpha^{2} 2 \delta|B(x)|+2 \alpha\|V\|_{\infty}\|\mathbb{1}\|_{2}\|g\|_{2}+\|V\|_{\infty}\|g\|_{2}^{2}\right] .
$$

For any $a, b \in \mathbb{R}$ and $\gamma>0$ we have the inequality $2 a b \leq \gamma a^{2}+b^{2} / \gamma$. Pick $a=\alpha\|V\|_{\infty}\|\mathbb{1}\|_{2}$ and $b=\|g\|_{2}$. Then we may further estimate (B.13) from above by

$$
\begin{aligned}
& -\eta\|g\|_{2}^{2}+\frac{1}{\kappa}\left[\alpha^{2} 2 \delta|B(x)|+\gamma \alpha^{2}\|V\|_{\infty}^{2}\|1\|_{2}^{2}+\|g\|_{2}^{2} / \gamma+\|g\|_{2}^{2}\right] \\
& =\|g\|_{2}^{2}\left[-\eta+\frac{1}{\kappa}\left(1+\frac{1}{\gamma}\right)\right]+\frac{1}{\kappa}\left[2 \delta+\gamma\|V\|_{\infty}^{2}\right] \alpha^{2}\|\mathbb{1}\|_{2}^{2},
\end{aligned}
$$

where we use that $\|1\|_{2}^{2}=|B(x)|$. Now pick $\gamma=2 \delta /\|V\|_{\infty}^{2}$ and note that, for $\kappa$ large enough so that $\frac{1}{\kappa}(1+1 / \gamma-4 \delta) \leq \eta$, we have $-\eta+\frac{1}{\kappa}(1+1 / \gamma) \leq 4 \frac{1}{\kappa} \delta$. Therefore, for $\kappa$ large enough we may estimate the right-most term in (B.14) from above by

$$
4 \frac{1}{\kappa} \delta\left[\|g\|_{2}^{2}+\alpha^{2}\|1\|_{2}^{2}\right]=4 \frac{1}{\kappa} \delta\|f\|_{2}^{2} .
$$

\section{References}

[1] S. Akkouche, The spectral bounds of the discrete Schrödinger operator, J. Funct. Anal. 259 (2010) 1443-1465.

[2] P. Erdös, On an elementary proof of some asymptotic formulas in the theory of partitions, Ann. Math. 43 (1942) 437-450.

[3] D. Erhard, F. den Hollander and G. Maillard, The parabolic Anderson model in a dynamic random environment: basic properties of the quenched Lyapunov exponent. To appear in Ann. Inst. Henri Poincaré Probab. Stat. 2013.

http://arXiv:1208.0330v2.

[4] J. Gärtner and F. den Hollander, Intermittency in a catalytic random medium, Ann. Probab. 34 (2006) 2219-2287.

[5] J. Gärtner, F. den Hollander and G. Maillard, Intermittency on catalysts, in: Trends in Stochastic Analysis (eds. J. Blath, P. Mörters and M. Scheutzow), London Mathematical Society Lecture Note Series 353, Cambridge University Press, Cambridge, 2009, pp. 235248. 
[6] J. Gärtner, F. den Hollander and G. Maillard, Quenched Lyapunov exponent for the parabolic Anderson model in a dynamic random environment, in: Probability in Complex Physical Systems. In honour of Erwin Bolthausen and Jürgen Gärtner (eds. J.-D. Deuschel, B. Gentz, W. König, M.-K. van Renesse, M. Scheutzow, U. Schmock), Springer Proceedings in Mathematics 11, Springer, 2012, Berlin, pp. 159-193.

[7] G.H. Hardy and S.A. Ramanujan, Asymptotic formulae in combinatory analysis, Proc. London Math. Soc. 17 (1918) 75-115.

[8] Y. Higuchi, T. Matsumoto and O. Ogurisu, On the spectrum of a discrete Laplacian on $\mathbb{Z}$ with finitely supported potential, Linear and Multilinear Algebra 59 (2011) 917-927.

[9] F. den Hollander. Large Deviations, Fields Institute Monograph 10, American Mathematical Society, Providence RI, 2000.

[10] H. Kesten and V. Sidoravicius, Branching random walks with catalysts, Electr. J. Prob. 8 (2003) 1-51.

[11] W. Kirsch, An invitation to random Schrödinger operators, http://arxiv.org/abs/0709.3707

[12] E.H. Lieb and M. Loss, Analysis (2nd. ed), AMS Graduate Studies 14, American Mathematical Society, Providence RI, 2001.

[13] A.R. Pruss, Symmetrisation, Green's functions, Harmonic Measures and Difference Equations, Doctoral dissertation, University of British Columbia, 1996. https://bearspace.baylor.edu/Alexander_Pruss/www/papers/index.html

[14] A.R. Pruss, Discrete convolution-rearrangement inequalities and the Faber-Krahn inequality on regular trees, Duke Math. J. 91 (1998) 463-514.

[15] M. Reed and B. Simon, Methods of Modern Mathematical Physics, Vol. 4, Analysis of Operators, Academic Press, Inc., San Diego, 1978.

[16] A. Rényi, Foundations of Probability, Holden-Day, Inc., San Francisco, CA 1970.

[17] A. Sahovic, New constants in discrete Lieb-Thirring inequalities for Jacobi matrices, J. Math. Sci. N.Y. 166 (2010) 319-327. 